\title{
Parameterization of single-scattering properties of snow
}

\author{
P. Räisänen ${ }^{1}$, A. Kokhanovsky ${ }^{2,3}$, G. Guyot ${ }^{4}$, O. Jourdan ${ }^{4}$, and T. Nousiainen ${ }^{1}$ \\ ${ }^{1}$ Finnish Meteorological Institute, P.O. Box 503, 00101 Helsinki, Finland \\ ${ }^{2}$ EUMETSAT, Eumetsat Allee 1, 64295 Darmstadt, Germany \\ ${ }^{3}$ Institute of Remote Sensing, University of Bremen, 28334 Bremen, Germany \\ ${ }^{4}$ Laboratoire de Météorologie Physique (LaMP) Université Blaise Pascal/CNRS/OPGC, 24 avenue des Landais, \\ 63177 Aubiére CEDEX, France
}

Correspondence to: P. Räisänen (petri.raisanen@fmi.fi)

Received: 16 December 2014 - Published in The Cryosphere Discuss.: 13 February 2015

Revised: 02 June 2015 - Accepted: 05 June 2015 - Published: 23 June 2015

\begin{abstract}
Snow consists of non-spherical grains of various shapes and sizes. Still, in many radiative transfer applications, single-scattering properties of snow have been based on the assumption of spherical grains. More recently, second-generation Koch fractals have been employed. While they produce a relatively flat phase function typical of deformed non-spherical particles, this is still a rather ad hoc choice. Here, angular scattering measurements for blowing snow conducted during the CLimate IMpacts of Short-Lived pollutants In the Polar region (CLIMSLIP) campaign at Ny Ålesund, Svalbard, are used to construct a reference phase function for snow. Based on this phase function, an optimized habit combination (OHC) consisting of severely rough (SR) droxtals, aggregates of SR plates and strongly distorted Koch fractals is selected. The single-scattering properties of snow are then computed for the OHC as a function of wavelength $\lambda$ and snow grain volume-to-projected area equivalent radius $r_{\mathrm{vp}}$. Parameterization equations are developed for $\lambda=0.199-2.7 \mu \mathrm{m}$ and $r_{\mathrm{vp}}=10-2000 \mu \mathrm{m}$, which express the single-scattering co-albedo $\beta$, the asymmetry parameter $g$ and the phase function $P_{11}$ as functions of the size parameter and the real and imaginary parts of the refractive index. The parameterizations are analytic and simple to use in radiative transfer models. Compared to the reference values computed for the $\mathrm{OHC}$, the accuracy of the parameterization is very high for $\beta$ and $g$. This is also true for the phase function parameterization, except for strongly absorbing cases $(\beta>0.3)$. Finally, we consider snow albedo and reflected radiances for the suggested snow optics parameterization, making comparisons to spheres and distorted Koch fractals.
\end{abstract}

\section{Introduction}

Snow grains are non-spherical and often irregular in shape. Still, in many studies, spherical snow grains have been assumed in radiative transfer calculations due to the convenience of using Mie theory. In fact, it has been shown that the spectral albedo of snow can be fitted by radiative transfer calculations under the assumption of spherical snow grains, when the effective snow grain size is considered an adjustable parameter (i.e. determined based on albedo rather than microphysical measurements) (Wiscombe and Warren, 1980; Grenfell et al., 1994; Aoki et al., 2000). Snow albedo parameterizations used in climate models and numerical weather prediction models are often semi-empirical and do not specify the snow grain shape (for some examples, see Wang and Zeng, 2010). However, in most (if not all) physically based albedo parameterizations that explicitly link the albedo to snow grain size, spherical snow grains are assumed (Flanner and Zender, 2005; Gardner and Sharp, 2010; Aoki et al., 2011).

It is, however, well known that the single-scattering properties (SSPs) of non-spherical particles, including the singlescattering albedo $\omega$, the phase function $P_{11}$ and the entire phase matrix $\mathbf{P}$, can differ greatly from those of spheres. ${ }^{1} \mathrm{~A}$ consequence of this is that the assumed shape of snow grains has a profound effect on the bidirectional reflectance distribution function (BRDF) of snow (Mischenko et al., 1999; Xie et al., 2006). Furthermore, Aoki et al. (2000) showed that the modelled BRDF of snow agreed better with observa-

\footnotetext{
${ }^{1}$ While symbols and abbreviations are introduced at their first appearance, they are also listed in Table A1.
} 
tions when, instead of the actual phase function for spheres, the Henyey-Greenstein (HG) phase function (Henyey and Greenstein, 1941) was assumed. The HG phase function is very smooth, while that of spheres features icebow and glory peaks not seen for real snow along with very low sideward scattering. Based on a comparison of a few shape models with phase function measurements for laboratory-generated ice crystals (Barkey et al., 2002), Kokhanovsky and Zege (2004) recommended, instead of spheres, the use of Gaussian random spheres (Muinonen et al., 1996; Nousiainen and Muinonen, 1999) or Koch fractals (Macke et al., 1996), which both exhibit a relatively featureless phase function. Since Gaussian random spheres have several free parameters while Koch fractals have none (except for the degree of distortion for randomized Koch fractals), Koch fractals were selected by Kokhanovsky and Zege (2004). Kokhanovsky et al. $(2005,2011)$ further demonstrated that the reflectance patterns computed for Koch fractals agreed reasonably well with actual measurements for snow. Subsequently, they have been used in several studies related to remote sensing of snow grain size and snow albedo (Lyapustin et al., 2009; Negi and Kokhanovsky, 2011; Kokhanovsky et al., 2011).

Other snow grain shape models have also been considered. Tanikawa et al. (2006) suggested the use of non-spherical ice particles with rough surfaces, specifically, cylindrical particles for new snow and prolate ellipsoids for old granular snow. These choices improved the agreement with observed angular reflectance patterns compared to the use of spheres. Jin et al. (2008) compared anisotropic reflectance factors computed using spheres, hexagonal plates, hexagonal columns and aggregates of columns with ground-based measurements in Antarctica, finding the best agreement for the aggregate model and the largest discrepancies for spheres. Furthermore, Zege et al. (2011) tested, in their retrieval algorithm of snow grain size and soot concentration in snow, a mixture of hexagonal columns and plates with rough surfaces.

Overall, while it is clear that spheres are not an ideal choice for modelling the SSPs of snow, it is less clear which non-spherical model should be used. Kokhanovsky and Zege (2004) noted that the final decision of the shape model should be made when in situ phase function measurements for snow become available. The present paper makes a step towards this direction. We employ angular scattering measurements for blowing snow performed with a polar nephelometer (Gayet et al., 1997) during the CLimate IMpacts of Short-Lived pollutants In the Polar region (CLIMSLIP) campaign at Ny Ålesund, Svalbard (Guyot et al., 2015), to construct a reference phase function for snow grains at the wavelength $\lambda=0.80 \mu \mathrm{m}$. This phase function is used to select a new shape model for snow, an "optimized habit combination" (OHC) consisting of severely rough (SR) droxtals, aggregates of SR plates and strongly distorted Koch fractals. The SSPs for the OHC are then computed as a function of wavelength and snow grain size, and parameterization equations are developed for the single-scattering co-albedo $\beta=1-\omega$, the asymmetry parameter $g$ and the phase function $P_{11}$. Such parameterizations are of substantial practical significance, as they greatly facilitate the use of the $\mathrm{OHC}$ in radiative transfer applications. We are not aware of any such previous parameterizations for representing the snow SSPs.

The outline of this paper is as follows. First, in Sect. 2, the models used to compute the SSPs of Koch fractals, Gaussian spheres and spheres are introduced along with the database of Yang et al. (2013) used for several other shapes. In Sect. 3, the reference phase function for snow is constructed. In Sect. 4, several shape models are compared in terms of their ability to reproduce the reference phase function, and the OHC is selected. In Sect. 5, the SSPs for the OHC are computed as a function of wavelength and snow grain size, and in Sect. 6 parameterization equations are developed. In Sect. 7, the snow SSP parameterization is applied to radiative transfer computations, and comparisons are made to spheres and Koch fractals. Finally, a summary is given in Sect. 8.

\section{Shape models and single-scattering data}

Here, several shape models are considered as candidates for representing the SSPs of snow. These include (1) secondgeneration Koch fractals, (2) Gaussian random spheres, (3) nine different crystal habits in the Yang et al. (2013) single-scattering database and, for comparison, (4) spheres. The snow grains are assumed to consist of pure ice (i.e. no impurities such as black carbon are included). The ice refractive index of Warren and Brandt (2008) is employed.

The SSPs (extinction cross section, single-scattering albedo, phase function and asymmetry parameter) of Koch fractals are simulated using the geometric optics code of Macke (1993) (see also Macke et al., 1996). Both regular and distorted Koch fractals are considered. A regular secondgeneration Koch fractal has 144 equilateral triangular surface elements. Distortion is simulated using a statistical approach, in which for each refraction-reflection event the normal of the crystal surface is tilted randomly around its original direction (Macke et al., 1996). The zenith (azimuth) tilt angle is chosen randomly with equal distribution between $\left[0, \theta^{\max }\right]$ $\left(\left[0,360^{\circ}\right]\right)$, where $\theta^{\max }$ is defined using a distortion parameter $t=\theta^{\max } / 90^{\circ}$. Values of $t=0$ (regular), $t=0.18$ (distorted) and $t=0.50$ (strongly distorted) are considered. The geometric optics solution consists of ray tracing and diffraction parts, which are combined as in Macke et al. (1996). For diffraction, the Fraunhofer (far-field) approximation is employed. Either 3 million (in Sect. 4) or 1 million (in Sect. 5) rays per case (i.e. crystal size, wavelength and degree of distortion) are used for the ray tracing part. Up to $p=12$ ray-surface interactions per initial ray are considered (see Sect. 3A in Macke, 1993).

The SSPs of Gaussian random spheres are computed with the geometric optics code of Muinonen et al. (1996). Details 
of the Gaussian random sphere shape model are discussed e.g. in Nousiainen and McFarquhar (2004). The shape of the particles is described in terms of three parameters: the relative $\mathrm{SD}$ of radius $\sigma$, the power-law index $v$ in the Legendre polynomial expansion of the correlation function of radius (the weight of the $n$th degree Legendre polynomial $P_{n}$ being $c_{n} \propto n^{-v}$ ), and the degree of truncation $n_{\max }$ for this polynomial expansion. In broad terms, increasing $\sigma$ increases the large-scale non-sphericity of the particle, while decreasing $v$ and increasing $n_{\max }$ adds small-scale structure to the particle shape. Four values were considered for $\sigma(0.15,0.20,0.25$ and 0.30), four for $v\left(1.5,2.0,2.5\right.$ and 3.0) and three for $n_{\max }$ $(15,25$ and 35$)$, which yields 48 parameter combinations. A total of 1 million rays with 1000 realizations of particle shape per case were employed in the ray tracing computations. Diffraction was computed by applying the Fraunhofer approximation to equivalent cross-section spheres.

Recently, Yang et al. (2013) published a comprehensive library of SSPs of non-spherical ice crystals, for wavelengths ranging from the ultraviolet to the far infrared, and for particle maximum dimensions $d_{\max }$ ranging from 2 to $10000 \mu \mathrm{m}$. The library is based on the Amsterdam discrete dipole approximation (Yurkin et al., 2007) for small particles (size parameter smaller than about 20) and improved geometric optics (Yang and Liou, 1998; Bi et al., 2009) for large particles. Here, single-scattering properties for nine ice particle habits in the Yang et al. (2013) database are used: droxtals, solid and hollow hexagonal columns, aggregates of 8 columns, plates, aggregates of 5 and 10 plates, and solid and hollow bullet rosettes. For each habit, the SSPs are provided for three degrees of particle surface roughness: completely smooth (CS), moderately rough (MR) and severely rough. The effect of roughness is simulated in a way that closely resembles the treatment of distortion for Koch fractals: the surface slope is distorted randomly for each incident ray, assuming a normal distribution of local slope variations with a SD of $0,0.03$ and 0.50 for the CS, MR and SR particles respectively in Eq. (1) of Yang et al. (2013). In fact, this approach does not represent any specific roughness characteristics but attempts instead to mimic the effects on SSPs due to non-pristine crystal characteristics in general (both roughness effects and irregularities).

For comparison, results are also shown for spheres. The SSPs of spheres are computed using a Lorenz-Mie code (de Rooij and van der Stap, 1984; Mischenko et al., 1999).

\section{Observation-based phase function for blowing snow}

We employ as a reference an observation-based phase function for blowing snow. The reference phase function was derived from ground-based measurements conducted during the CLIMSLIP field campaign at Ny Ålesund, Svalbard (Guyot et al., 2015), on 23 and 31 March 2012. The blowing snow case on 23 March was preceded by heavy snow- fall on 22 March, ending during the night of 23 March. The last snowfall before 31 March blowing snow case occurred on 29 March. Consequently, the case of 23 March represents essentially new snow, while on 31 March some snow metamorphism had occurred, and the snowpack was probably denser (although snow density was not measured). The near-surface air temperature ranged from -5 to $-9{ }^{\circ} \mathrm{C}$ during the 23 March case and from -18 to $-20^{\circ} \mathrm{C}$ during 31 March. Correspondingly, the wind speeds ranged from 1 to $9 \mathrm{~m} \mathrm{~s}^{-1}$ on 23 March (median value $4 \mathrm{~m} \mathrm{~s}^{-1}$ ) and from 5 to $8 \mathrm{~m} \mathrm{~s}^{-1}$ on 23 March (median value $7 \mathrm{~m} \mathrm{~s}^{-1}$ ). Mainly cloudy conditions prevailed on 23 March, while 31 March was cloud free. The phase functions discussed below are averages over the entire blowing snow events, which lasted for approximately $10 \mathrm{~h}$ (8-18 UTC) on 23 March and $12 \mathrm{~h}$ (12-24 UTC) on 31 March.

The angular scattering coefficient $\Psi\left(\theta_{\mathrm{s}}\right)\left[\mu \mathrm{m}^{-1} \mathrm{sr}^{-1}\right]$ of blowing snow was measured with a polar nephelometer (PN; Gayet et al., 1997; Crépel et al., 1997) on 23 and 31 March 2012 at 31 scattering angles in the $15^{\circ} \leq \theta_{\mathrm{s}} \leq 162^{\circ}$ range at a nominal wavelength of $\lambda=0.80 \mu \mathrm{m}$. The corresponding phase function $P_{11}\left(\theta_{\mathrm{s}}\right)$ was obtained by normalizing $\Psi\left(\theta_{\mathrm{s}}\right)$ by the volume extinction coefficient $\sigma_{\text {ext }}$ :

$P_{11}\left(\theta_{\mathrm{s}}\right)=4 \pi \frac{\Psi\left(\theta_{\mathrm{s}}\right)}{\sigma_{\mathrm{ext}}}$.

Here $\sigma_{\text {ext }}$ was estimated from the PN data following Gayet et al. (2002), with a quoted accuracy of $25 \%$.

The derived phase functions are shown in Fig. 1a. There are only minor differences between the 23 and 31 March cases. In both cases $P_{11}$ decreases sharply from 15 to $50^{\circ}$, then more gradually until $127^{\circ}$. At larger scattering angles $P_{11}$ increases slightly with a local maximum around $145^{\circ}$ (discussed below). Hereafter, the average over the two cases is used as a reference for the modelled phase functions:

$P_{11}^{\mathrm{ref}}=0.5 \cdot\left(P_{11}^{23 \text { March }}+P_{11}^{31 \text { March }}\right)$.

In Fig. $1 \mathrm{~b}, P_{11}^{\text {ref }}$ is compared with three other phase functions: a non-precipitating cirrus case over Southern France in the CIRRUS'98 experiment (Durand et al., 1998) (discussed in Jourdan et al., 2003) and two phase functions for glaciated parts of nimbostratus over Svalbard in the ASTAR 2004 experiment, corresponding to clusters 6 and 7 in Jourdan et al. (2010). These phase functions were derived from raw PN data using a statistical inversion scheme (Jourdan et al., 2003, 2010). Perhaps as expected, the blowing snow phase function $P_{11}^{\text {ref }}$ is generally closer to the glaciated nimbostratus phase functions than to the cirrus phase function. In particular, at sideward angles between roughly 55 and $135^{\circ}, P_{11}^{\text {ref }}$ falls mostly between the two nimbostratus phase functions, while the cirrus phase function exhibits somewhat smaller values. The smallest $P_{11}$ in the cirrus and nimbostratus cases occurs at $\theta_{\mathrm{s}}=120^{\circ}$, as compared with $\theta_{\mathrm{s}}=127^{\circ}$ for $P_{11}^{\text {ref }}$. All four 

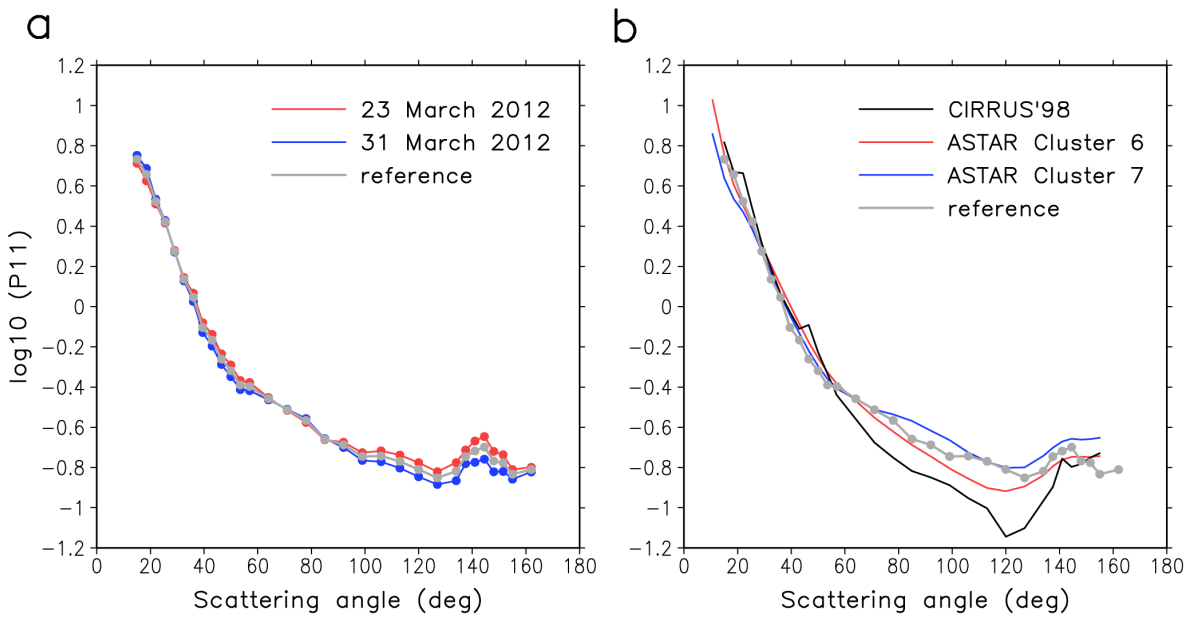

Figure 1. (a) Phase function of blowing snow as derived from the CLIMSLIP data on 23 March 2012 (red) and on 31 March 2012 (blue). The reference phase function $P_{11}^{\text {ref }}$ (grey) was defined as the average of the 23 and 31 March cases. (b) Comparison of $P_{11}^{\text {ref }}$ with phase functions for non-precipitating cirrus (CIRRUS'98, black line) and glaciated Arctic nimbostratus (ASTAR clusters 6 and 7, red and blue lines).

phase functions then increase until $\theta_{\mathrm{s}} \approx 140^{\circ}$, after which the nimbostratus and cirrus phase functions become quite flat. In contrast, $P_{11}^{\text {ref }}$ shows a local maximum around $\theta_{\mathrm{s}} \approx 145^{\circ}$.

The origin of the maximum at $\theta_{\mathrm{s}} \approx 145^{\circ}$ is not clear. While it may, in principle, be caused by scattering by snow grains, this feature is neither captured by any of the particle shapes considered in this study nor present in phase functions measured for laboratory-generated ice crystals in Barkey et al. (2002) and Smith et al. (2015). Rather, it falls between the icebow peak for spherical ice particles near $135^{\circ}$ and a maximum seen for many pristine hexagonal shapes at $150-155^{\circ}$ (see Fig. 3). Curiously, this feature coincides with the scattering maximum of small water droplets with a $\sim 10 \mu \mathrm{m}$ diameter at $140-145^{\circ}$. However, water droplets seem like an implausible explanation, since the conditions at the measurement site were subsaturated with respect to liquid water (the relative humidity being roughly $92-95 \%$ on 23 March and $79-87 \%$ on 31 March), and especially the 31 March case was quite cold. Yet the $145^{\circ}$ feature is clearly visible in the measured phase function in both cases. Finally, we cannot discount the possibility that inaccuracy in the PN angular scattering measurements influences this feature. Shcherbakov et al. (2006) report relative accuracy of scattered intensities of $3-5 \%$ between 15 and $141^{\circ}$, but degrading to $30 \%$ for $162^{\circ}$, for an experimental setup with low extinction. Thus the phase function derived from the PN measurements is, overall, less reliable near the backscattering direction than in near-forward and side-scattering directions.

Whether the phase function feature at $145^{\circ}$ is an artifact or a real feature caused by scattering by snow should be resolved through further measurements, preferably using some alternative technique. However, in either case, it has only a small impact on the snow SSP parameterizations derived in this paper. This detail cannot be captured by any of the shape models considered, so it is not present in the parameterized phase functions. Its influence on the asymmetry parameter is also modest. Even a complete elimination of the maximum by linear interpolation of $P_{11}^{\text {ref }}$ between the minima at 127 and $155^{\circ}$ would increase $g$ by only $\approx 0.007$.

The size distribution of blowing snow was measured with the Cloud Particle Imager (CPI) instrument (Lawson et al., 2001). The CPI registers particle images on a solid state, one million pixel digital charge-coupled device (CCD) camera by freezing the motion of the particle using a $40 \mathrm{~ns}$ pulsed, high power laser diode. Each pixel in the CCD camera array has an equivalent size in the sample area of $2.3 \mu \mathrm{m}$. In the present study, the minimum size for the CPI's region of interest is set up to 10 pixels. Therefore particles with sizes ranging approximately from $25 \mu \mathrm{m}$ to $2 \mathrm{~mm}$ are imaged.

Figure 2a shows examples of particles imaged by the CPI on 31 March 2012. While some needle-shaped crystals can be spotted, many of the particles are irregular, which also applies to the 23 March 2012 case. It is also noted that many of the particles show rounded edges, possibly related to sublimation during snow metamorphosis. Size distributions derived from the CPI data are shown in Fig. 2b. A lognormal distribution was fitted to the data (averaged over the 23 and 31 March cases):

$n\left(d_{\mathrm{p}}\right)=\frac{1}{\sqrt{2 \pi} \ln \sigma_{\mathrm{g}} d_{\mathrm{p}}} \exp \left[-\frac{\left(\ln d_{\mathrm{p}}-\ln d_{\mathrm{p}, 0}\right)^{2}}{2 \ln ^{2} \sigma_{\mathrm{g}}}\right]$.

Here, $d_{\mathrm{p}}$ is the projected-area equivalent diameter of the particles, $d_{\mathrm{p}, 0}=187 \mu \mathrm{m}$ is the median diameter and $\sigma_{\mathrm{g}}=1.62$ the geometric SD. This size distribution was used for all shape models when comparing the modelled phase functions with $P_{11}^{\text {ref }}$. Since absorption is weak at $\lambda=0.80 \mu \mathrm{m}$ and the particles are much larger than the wavelength, the modelled $P_{11}$ is only weakly sensitive to the size distribution employed 

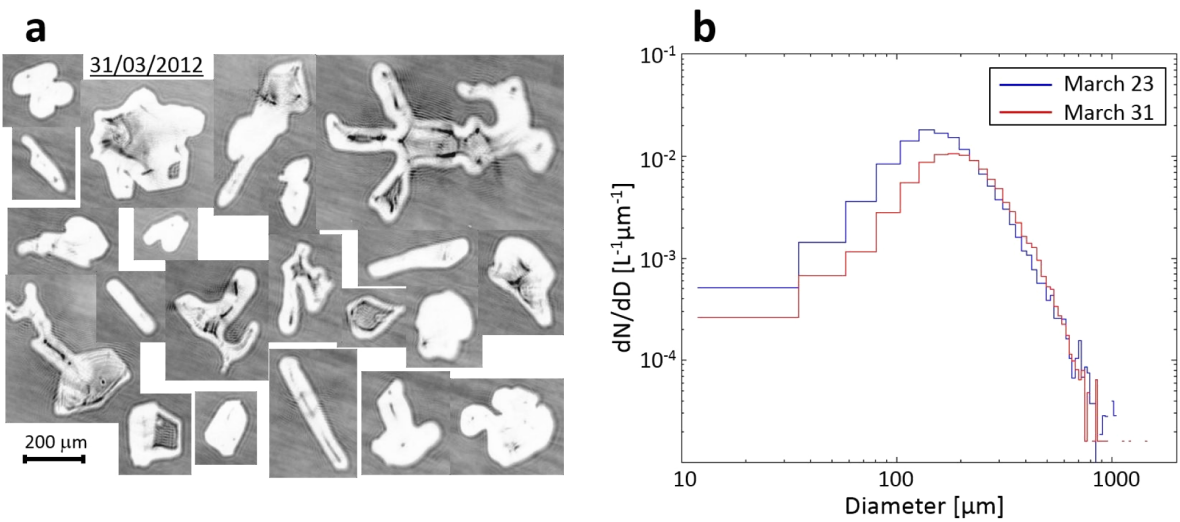

Figure 2. (a) Examples of snow grains imaged by the CPI instrument on 31 March 2012 and (b) size distributions for both the 23 and 31 March cases.

if the shape of the snow grains is independent of size. This holds true for spheres, Gaussian spheres, Koch fractals, droxtals and the three aggregate habits in the Yang et al. (2013) database. However, for solid and hollow hexagonal columns, plates as well as solid and hollow bullet rosettes, the crystal geometry is a function of size, with some influence on $P_{11}$ (see end of Sect. 4 for more discussion).

\section{Selecting a shape model for snow optics}

The purpose of this section is to select a shape model of snow for use in Sects. 5 to 7. The phase function for blowing snow from the CLIMSLIP campaign, as defined by Eq. (2), is used as a reference. It is emphasized that the approach is deliberately pragmatic: we do not attempt to model the scattering based on the shapes of the observed snow grains; rather we try to develop an equivalent microphysical model for representing the SSPs. Previously, the choice of Koch fractals for approximating the scattering by snow (Kokhanovsky and Zege, 2004) was likewise based on phase function data only. Furthermore, our approach is conceptually analogous to the widely used practice of modelling the SSPs of irregular dust particles. Instead of considering the actual dust particle shapes, shape distributions of spheroids are used operationally in a variety of applications (Dubovik et al., 2006, 2011; Levy et al., 2007), as they have been found to reasonably mimic scattering by dust. In contrast, current state-of-the-art models for ice cloud SSPs include ice crystal habit distributions parameterized as a function of crystal size, based on in situ microphysical observations (Baum et al., 2005, 2011; Hong et al., 2009). In principle, it would be desirable to use this approach also for snow to provide a more direct link between the actual snow grain shapes and those assumed in the parameterization and to account for changes in snow grain shape with size, which we currently neglect. This would require, first, the analysis and subsequent parameterization of snow grain shape distributions as a function of size and, second, the computation and parameterization of the respective SSPs. The main reason why we have not attempted this approach in the current work is that a very large fraction of the particles in blowing snow (and snow on ground) are irregular, more than $80 \%$ according to manual classification of CPI images (Guyot et al., 2015) (see also Fig. 2a), and cannot be unambiguously associated with habits considered e.g. in the database of Yang et al. (2013).

To provide a quantitative measure for the agreement between the modelled and reference phase functions ( $P_{11}^{\text {model }}$ and $P_{11}^{\text {ref }}$ respectively) we define a cost function as the rootmean-square (rms) error of the logarithm of phase function:

$\operatorname{cost}=\sqrt{\frac{\int_{15^{\circ}}^{162^{\circ}}\left(\ln P_{11}^{\text {model }}-\ln P_{11}^{\text {ref }}\right)^{2} \sin \theta_{\mathrm{s}} \mathrm{d} \theta_{\mathrm{s}}}{\int_{15^{\circ}}^{162^{\circ}} \sin \theta_{\mathrm{s}} \mathrm{d} \theta_{\mathrm{s}}}}$.

To start with, the phase function for single crystal shapes is compared with $P_{11}^{\text {ref }}$ in Fig. 3. To be consistent with the CLIMSLIP observations, the phase function is computed at $\lambda=0.80 \mu \mathrm{m}$, and it is integrated over the size distribution defined by Eq. (3). Several points can be noted.

First, unsurprisingly, the phase function for spheres agrees poorly with the observations (Fig. 3a). In particular, sideward scattering is underestimated drastically, and there is a strong icebow peak at $\theta_{\mathrm{s}}=134^{\circ}$, which is not seen in $P_{11}^{\text {ref }}$.

Second, for second-generation Koch fractals (Fig. 3b), the agreement with $P_{11}^{\text {ref }}$ is considerably better than for spheres. The main features of the phase function are similar for regular and distorted Koch fractals. However, the regular Koch fractal's phase function exhibits several sharp features specific to the tetrahedral geometry which are not observed in $P_{11}^{\text {ref }}$. The distorted Koch fractals' versions are more consistent with the measurements even though marked deviations from $P_{11}^{\text {ref }}$ are still present. Scattering is underestimated between 15 and $30^{\circ}$ and overestimated between 45 and $100^{\circ}$. 

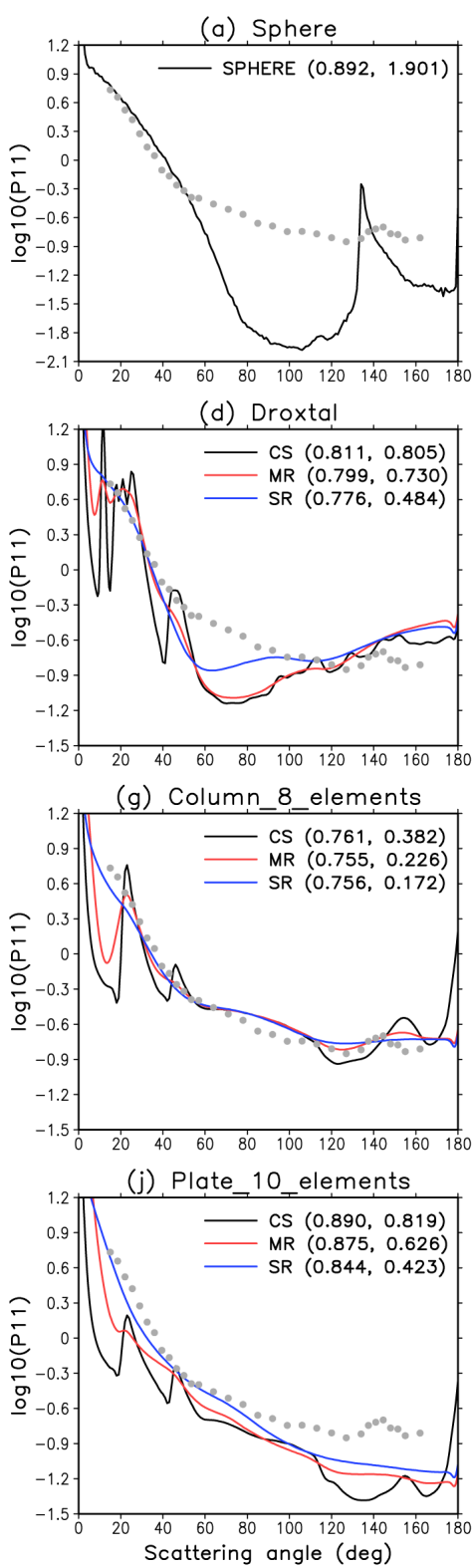

(b) Koch fractal

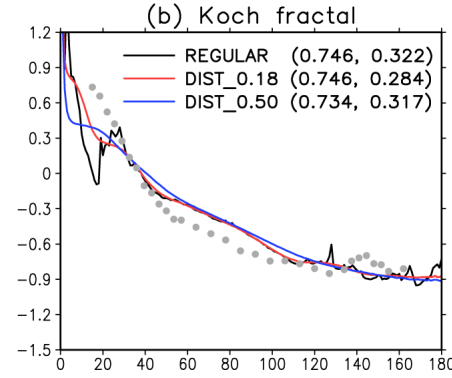

(e) Solid column

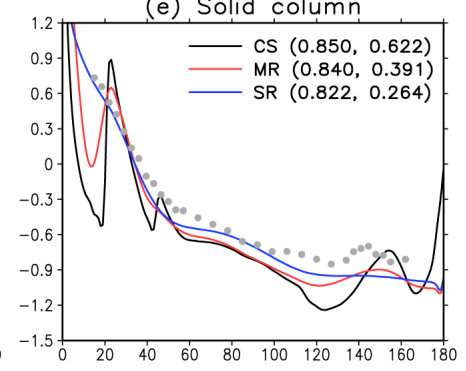

(h) Plate

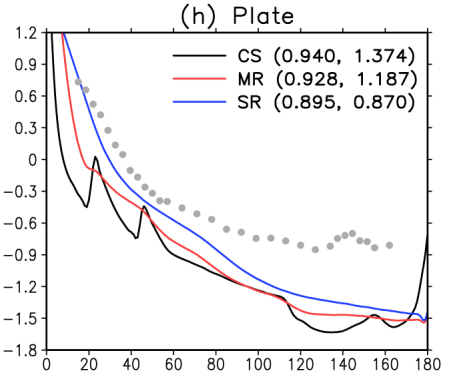

(k) Solid bullet rosette

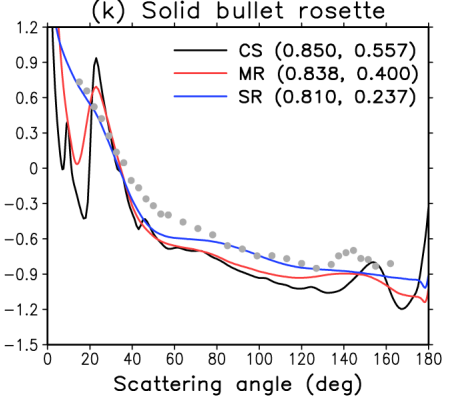

(c) Gaussian sphere

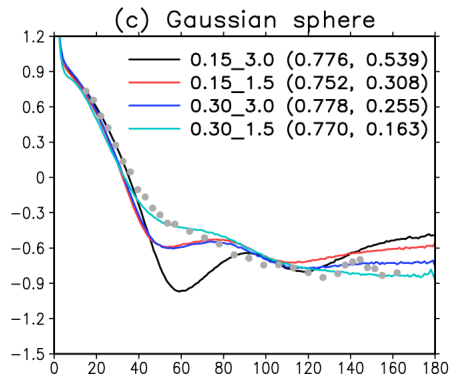

(f) Hollow column

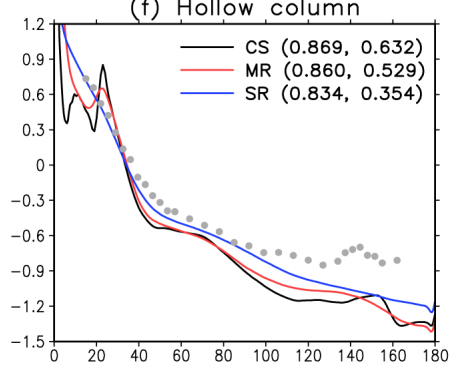

(i) Plate_5_elements

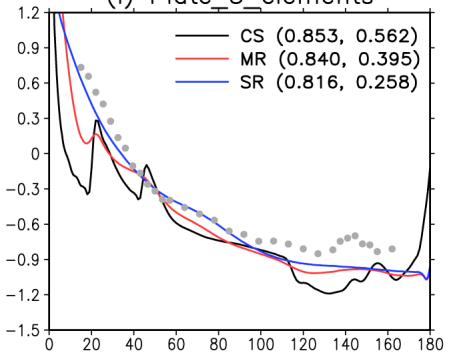

(I) Hollow bullet rosette

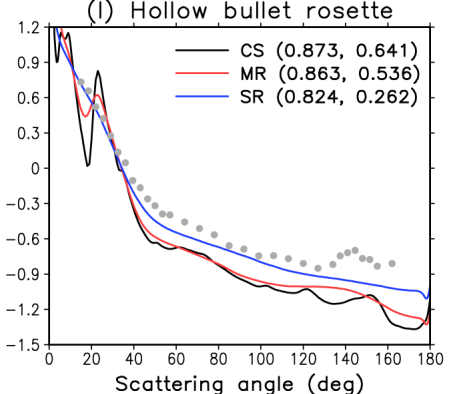

Figure 3. Comparison of phase function for various shape models with the reference phase function derived from CLIMSLIP data ( $P_{11}^{\text {ref }}$ shown with grey dots in each panel). (a) Spheres, (b) regular and distorted second-generation Koch fractals (with distortion parameters $t=0.18$ and $t=0.50)$, (c) four realizations of Gaussian spheres and (d-l) nine habits in the Yang et al. (2013) database. For each habit, the phase function was averaged over the size distribution defined by Eq. (3). In the figure legends, the two numbers in parentheses give the asymmetry parameter and the cost function defined by Eq. (4) respectively. For the Gaussian spheres in (c), the notation indicates the shape parameters (e.g. for 0.15_3.0, $\sigma=0.15$ and $v=3.0 ; n_{\max }$ was fixed at 15). For the Yang et al. (2013) habits in (d-l), CS, MR and SR refer to particles with completely smooth surface, moderate surface roughness and severe surface roughness respectively.

Also, the gradient of $P_{11}$ in the backscattering hemisphere is consistently negative, while $P_{11}^{\text {ref }}$ rather increases slightly between 127 and $162^{\circ}$. Overestimated sideward scattering by Koch fractals has been previously noted in the context of cirrus clouds (Francis et al., 1999) and in a comparison with a measured phase function for laboratory-generated ice crystals (Fig. 3 in Kokhanovsky and Zege, 2004).
Third, for Gaussian spheres, the level of agreement with $P_{11}^{\text {ref }}$ depends on the shape parameters chosen. Four cases out of the 48 considered are shown in Fig. 3c (for all of these $n_{\max }=15$, but the general features for $n_{\max }=25$ and $n_{\max }=35$ are similar). For example, for the parameter values $\sigma=0.15$ and $\nu=3.0$, which are close to those estimated from shape analysis of small quasi-spherical ice crystals in cirrus clouds in Nousiainen et al. (2011), the deviations 

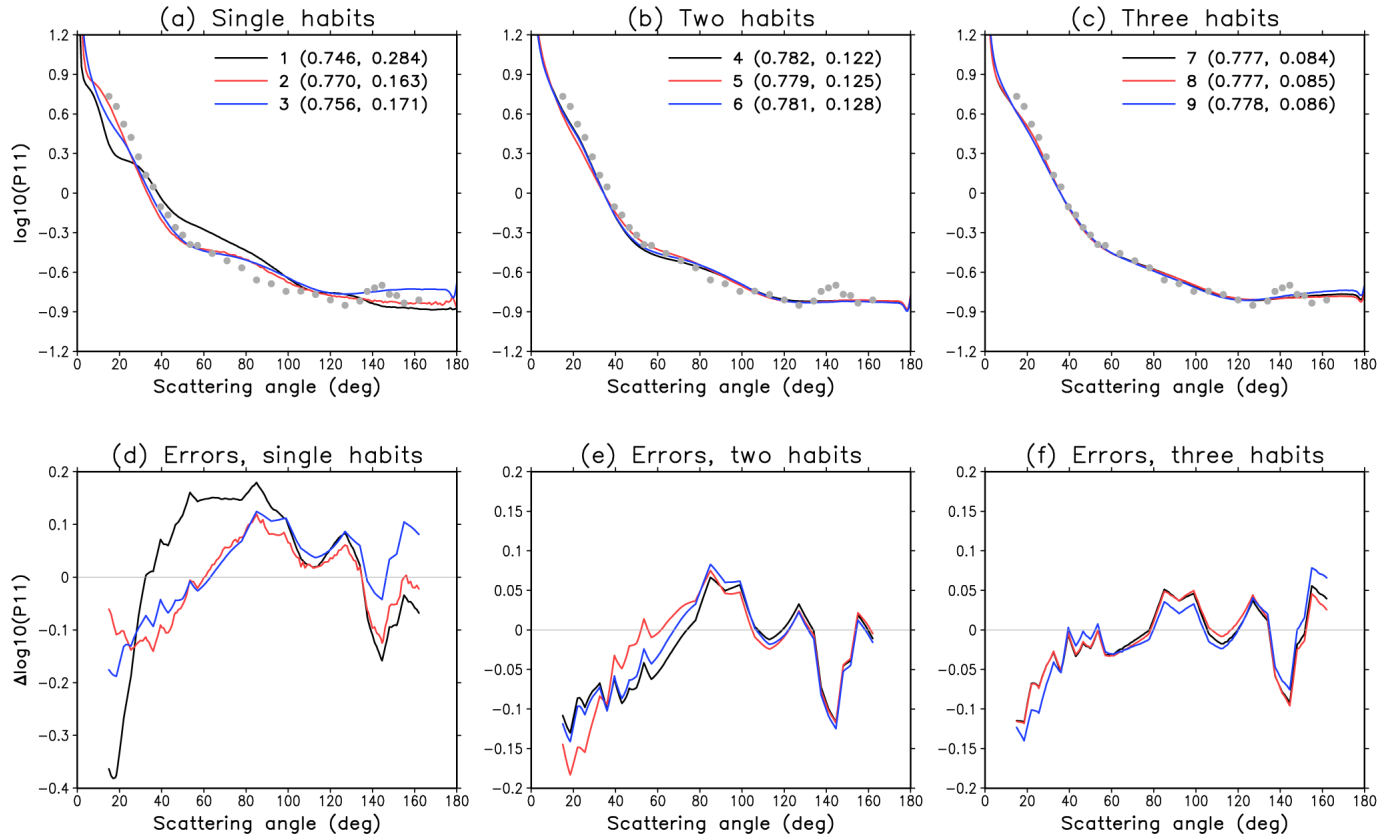

Figure 4. Comparison of modelled phase functions with the reference phase function $\left(P_{11}^{\text {ref }}\right.$ shown with grey dots in a-c). (a) Selected singlehabit cases: $1=$ distorted Koch fractals with $t=0.18 ; 2=$ Gaussian spheres with $\sigma=0.30, v=1.5$ and $n_{\max }=15$; and $3=$ aggregates of eight severely rough (SR) columns. (b) Best combinations of two habits: $4=$ aggregates of eight SR columns and SR hollow bullet rosettes (weights 0.61 and 0.39); $5=$ aggregates of eight SR columns and aggregates of five SR plates (weights 0.61 and 0.39 ); and $6=$ aggregates of eight SR columns and SR hollow columns (weights 0.68 and 0.32). (c) Best combinations of three habits: $7=$ SR droxtals, SR hollow columns and distorted Koch fractals $(t=0.50)$ (weights $0.32,0.30$ and 0.38 ); $8=$ SR droxtals, SR hollow bullet rosettes and distorted Koch fractals $(t=0.50)$ (weights $0.26,0.36$ and 0.38$)$; and $9=$ SR droxtals, aggregates of 10 SR plates and distorted Koch fractals $(t=0.50)$ (weights $0.36,0.26$ and 0.38 ). In the legends in $(\mathbf{a}-\mathbf{c})$, the two numbers in parentheses give the asymmetry parameter and the cost function defined by Eq. (4) respectively. (d-f) show the corresponding differences from $P_{11}^{\text {ref }}$.

from $P_{11}^{\text {ref }}$ are substantial. The phase function features undesirable large-scale oscillations and, in particular, scattering at $\theta_{\mathrm{s}} \approx 45-75^{\circ}$ is underestimated substantially. Best agreement with $P_{11}^{\text {ref }}$ is obtained in the case $\sigma=0.30, v=0.15$, which features both pronounced large-scale non-sphericity and small-scale structure in the particle shape. The sideward scattering is overestimated (mainly between 70 and $100^{\circ}$ ), but the cost function (0.163) is clearly smaller than that for distorted Koch fractals (0.284) and is, in fact, the smallest among all single-habit shape models considered.

Fourth, regarding the habits in the Yang et al. (2013) database (Fig. 3d-1), both visual inspection and the cost function values indicate that the agreement with $P_{11}^{\text {ref }}$ improves with increasing particle surface roughness. While completely smooth and, in many cases, moderately rough particles exhibit halo peaks, for severely rough particles the phase function is quite smooth and featureless, as is $P_{11}^{\text {ref }}$. It is further seen that, in general, increasing the roughness increases sideward scattering and reduces the asymmetry parameter. While none of the habits considered provides perfect agreement with $P_{11}^{\text {ref }}$, the cost function is smallest for the aggregate of eight columns (0.172).
Since none of the individual shape models agrees fully satisfactorily with $P_{11}^{\text {ref }}$, we considered combinations of two or three shapes. We thus use

$P_{11}^{\text {model }}=\sum_{j=1}^{n} w_{j} P_{11}^{j}$,

where $n=2$ or $n=3$ is the number of shapes in a combination and $P_{11}^{j}$ is the phase function for shape $j$, integrated over the size distribution (Eq. 3) for each shape separately. Thus, the potential dependence of snow grain shapes on their size is not considered here. For each combination of shapes considered, the optimal weight factors $w_{j}$ were searched by minimizing the cost function (Eq. 4), subject to the conditions that all $w_{j}$ are non-negative and their sum equals 1 . Since pristine particles and even moderately rough particles feature halo peaks (or an icebow peak in the case of spheres), which are absent in $P_{11}^{\text {ref }}$, the following groups of habits are considered: distorted Koch fractals, Gaussian spheres and severely rough particles in the Yang et al. (2013) database.

Figure 4 illustrates a comparison with $P_{11}^{\text {ref }}$ for three singlehabit cases (Fig. 4a and d) (the best Koch fractal case, the best Gaussian sphere case and the best case with Yang et al. 
Table 1. List of best three-habit combinations. $w_{1}, w_{2}$ and $w_{3}$ are the weights (i.e. fractional contributions to projected area) of each habit, "cost" is the cost function, $g$ the asymmetry parameter and $\xi$ a non-dimensional absorption parameter defined by Eq. (8). SR refers to severely rough particles, and $t$ is the distortion parameter for second-generation Koch fractals. The "optimized habit combination" (OHC) is highlighted with italic font.

\begin{tabular}{lcccclccc}
\hline$w_{1}$ & habit & $w_{2}$ & habit & $w_{3}$ & habit & cost & $g$ & $\xi$ \\
\hline 0.38 & fractal $(t=0.50)$ & 0.32 & SR droxtal & 0.30 & SR hollow column & 0.084 & 0.777 & 1.64 \\
0.38 & fractal $(t=0.50)$ & 0.26 & SR droxtal & 0.36 & SR hollow bullet rosette & 0.085 & 0.777 & 1.65 \\
0.38 & fractal $(t=0.50)$ & 0.36 & SR droxtal & 0.26 & aggregate (10 SR plates) & 0.086 & 0.778 & 1.62 \\
0.30 & fractal $(t=0.50)$ & 0.34 & SR droxtal & 0.36 & aggregate (5 SR plates) & 0.086 & 0.778 & 1.60 \\
0.46 & fractal $(t=0.50)$ & 0.36 & SR droxtal & 0.18 & SR plate & 0.087 & 0.778 & 1.66 \\
0.38 & fractal $(t=0.50)$ & 0.28 & SR droxtal & 0.34 & SR solid column & 0.090 & 0.776 & 1.63 \\
0.42 & fractal $(t=0.18)$ & 0.26 & SR droxtal & 0.32 & SR hollow bullet rosette & 0.095 & 0.779 & 1.66 \\
0.42 & fractal $(t=0.18)$ & 0.32 & SR droxtal & 0.26 & SR hollow column & 0.095 & 0.778 & 1.65 \\
0.34 & fractal $(t=0.18)$ & 0.32 & SR droxtal & 0.34 & aggregate (5 SR plates) & 0.096 & 0.779 & 1.61 \\
0.42 & fractal $(t=0.18)$ & 0.34 & SR droxtal & 0.24 & aggregate (10 SR plates) & 0.098 & 0.780 & 1.63 \\
\hline
\end{tabular}

(2013) particles), the best three two-habit cases (Fig. 4b and e) and the best three three-habit cases (Fig. 4c and f) as defined in terms of the cost function. As expected, the agree-

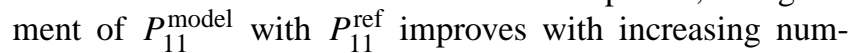
ber of shapes in the combination. The best three-habit cases follow $P_{11}^{\text {ref }}$ quite faithfully, though slightly underestimating $P_{11}^{\text {ref }}$ in near-forward directions and not capturing the details of $P_{11}^{\text {ref }}$ near $\theta_{\mathrm{s}}=145^{\circ}$. Furthermore, it is seen that the best three-habit combinations produce nearly identical $P_{11}$, agreeing even better with each other than with $P_{11}^{\text {ref }}$.

The relationship between the asymmetry parameter $g$ and the cost function is considered in Fig. 5, where all singlehabit cases and combinations of two or three habits are included. While high values of cost function can occur at any $g$, the lowest values $(<0.10)$ always occur for three-habit combinations with $0.775<g<0.78$. This supports a best estimate of $g \approx 0.78$ for snow at $\lambda=0.80 \mu \mathrm{m}$, of course subject to the assumption that the measurements for blowing snow used to construct $P_{11}^{\text {ref }}$ are also representative of snow on the ground.

The three-habit combinations with cost function below 0.1 are listed in Table 1. All of them include SR droxtals and either strongly distorted $(t=0.50)$ or distorted $(t=0.18)$ Koch fractals, but the third habit included in the combinations varies from case to case. The differences in cost function and asymmetry parameter between the best habit combinations are very small, which makes the choice of a single "best" habit combination for representing the SSPs of snow somewhat arbitrary. For further use in representing the SSPs as a function of wavelength and size, we select the following habit combination: $36 \%$ of SR droxtals, $26 \%$ of aggregates of $10 \mathrm{SR}$ plates and $38 \%$ strongly distorted secondgeneration Koch fractals $(t=0.50)$, where the weights refer to fractional contributions to the projected area. This habit combination is represented with a blue line in Fig. $4 \mathrm{c}$ and $\mathrm{f}$ and is marked with an arrow in Fig. 5. Hereafter, this habit combination will be referred to as the "optimized habit com-

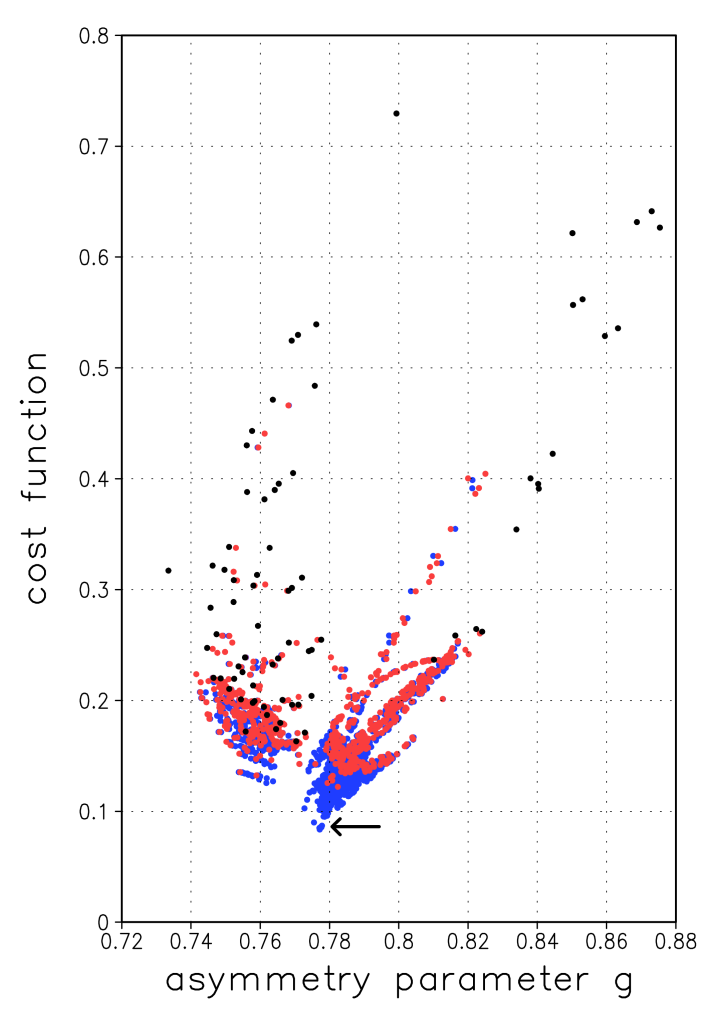

Figure 5. A scatter plot of asymmetry parameter vs. cost function (Eq. 4) for single habits (black dots), combinations of two habits (red dots) and combinations of three habits (blue dots). The "optimized habit combination" selected for parameterization of snow single-scattering properties is marked with an arrow. Note that some single-habit cases fall outside the range plotted here. These include spheres for which cost $=1.90$ and $g=0.892$.

bination". The primary reason why we selected this OHC rather than either of the first two habit combinations in Table 1 , which have a marginally lower cost function, is that these habit combinations include either hollow columns or 
bullet rosettes. For these habits (unlike aggregates of plates), the particle geometry assumed in the Yang et al. (2013) database depends on particle size, with the aspect ratio of the crystals increasing with their length. However, due to snow metamorphosis on ground, size-shape relationships based on crystal growth in ice clouds are most likely not valid for snow. Therefore, we considered it better to use a crystal geometry that is independent of size. This also helps to keep the SSP parameterization simpler.

\section{Snow single-scattering properties as a function of size and wavelength}

The SSPs, including the extinction efficiency $Q_{\text {ext }}$, singlescattering co-albedo $\beta$, asymmetry parameter $g$ and scattering phase function $P_{11}\left(\theta_{\mathrm{s}}\right)$ were determined for the $\mathrm{OHC}$ for 140 wavelengths between 0.199 and $3 \mu \mathrm{m}$ and for 48 particle sizes between 10 and $2000 \mu \mathrm{m}$. Here, the size is defined as the volume-to-projected area equivalent radius $r_{\mathrm{vp}}=0.75 \mathrm{~V} / \mathrm{P}$. As stated above, the OHC consists of SR droxtals, aggregates of $10 \mathrm{SR}$ plates and strongly distorted Koch fractals. The SSPs for droxtals and aggregates of plates were taken from the Yang et al. (2013) database (interpolated to fixed values of $r_{\mathrm{vp}}$ ) while those of Koch fractals were computed using the geometric optics code of Macke (1993), as explained in Sect. 2. Four caveats should be noted:

1. Due to problems associated with the truncation of numerical results to a finite number of digits ( $\mathrm{P}$. Yang, personal communication, 2013), the values of $\beta$ in the Yang et al. (2013) database are unreliable in cases of very weak absorption. To circumvent this issue, it was assumed that in cases of weak absorption $(\beta<0.001$ for Koch fractals), the values for droxtals and aggregates of plates may be approximated as

$$
\begin{aligned}
& \beta_{\text {droxtal }}\left(\lambda, r_{\mathrm{vp}}\right)=0.943 \beta_{\text {fractal }}\left(\lambda, r_{\mathrm{vp}}\right), \\
& \beta_{\text {aggregate }}\left(\lambda, r_{\mathrm{vp}}\right)=0.932 \beta_{\text {fractal }}\left(\lambda, r_{\mathrm{vp}}\right) .
\end{aligned}
$$

Here the scaling factors were determined as $\overline{\beta_{\text {droxtal }}} / \overline{\beta_{\text {fractal }}}$ and $\overline{\beta_{\text {aggregate }}} / \overline{\beta_{\text {fractal }}}$, where the overbar refers to averages over the cases in which $0.001<\beta_{\text {fractal }}<0.01$ and the size parameter $x=2 \pi r_{\mathrm{vp}} / \lambda>100$.

2. While the largest maximum dimension for particles in the Yang et al. (2013) database is $10000 \mu \mathrm{m}$ for all habits, the corresponding maximum values of $r_{\mathrm{vp}}$ are smaller and depend on the habit. For droxtals, $r_{\mathrm{vp}, \max }=4218 \mu \mathrm{m}$, while for the aggregates of 10 plates, it is only $r_{\mathrm{vp}, \max }=653 \mu \mathrm{m}$. Thus, to extend the SSPs for the OHC to sizes up to $r_{\mathrm{vp}}=2000 \mu \mathrm{m}$, we extrapolated the SSPs for the aggregates of plates based on how the SSPs depend on size for Koch fractals. See Appendix A for details.
3. The SSPs for Koch fractals were computed using a geometric optics code, which means that the accuracy deteriorates somewhat in cases with smaller size parameters (typically for $x<100$ ). This issue pertains mainly to small snow grains at near-IR wavelengths (e.g. for $\lambda=2.5 \mu \mathrm{m}, x=100$ corresponds to $\left.r_{\mathrm{vp}} \approx 40 \mu \mathrm{m}\right)$.

4. Lastly but importantly, since the OHC was selected based on measurements at a single wavelength $\lambda=0.80 \mu \mathrm{m}$ for only two cases, there is no guarantee that it represents the snow SSPs equally well at other wavelengths, or for all snow grain sizes.

Figure 6 compares wavelength-dependent SSPs for the OHC with those for two shape assumptions previously used in modelling snow optics: spheres and Koch fractals (distorted Koch fractals with $t=0.18$ were selected for this comparison; this is close though not identical to the shape assumption used by Kokhanovsky et al., 2011). Two monodisperse cases are considered, with $r_{\mathrm{vp}}=50 \mu \mathrm{m}$ and $r_{\mathrm{vp}}=1000 \mu \mathrm{m}$. For all three habits, the asymmetry parameter $g$ (Fig. 6a) and the single-scattering co-albedo $\beta$ (Fig. 6b) show well-known dependencies on particle size and wavelength. Thus, $g$ is largely independent of both $\lambda$ and $r_{\mathrm{vp}}$ in the visible region where $\beta$ is very small. In the near-IR region, $\beta$ increases with increasing imaginary part $m_{\mathrm{i}}$ of the refractive index and with increasing particle size. With increasing $\beta$, the fractional contribution of diffraction to the phase function increases, which results in larger values of $g$ (e.g. Macke et al., 1996). The most striking differences between the three shape assumptions occur for the asymmetry parameter, especially in the visible region, where $g \approx 0.89$ for spheres, $g \approx 0.74$ for distorted Koch fractals and $g \approx 0.77-0.78$ for the $\mathrm{OHC}$. The values of $\beta$ for the $\mathrm{OHC}$ are also intermediate between the two single-shape cases: larger than those for spheres (except for $r_{\mathrm{vp}}=1000 \mu \mathrm{m}$ at the strongly absorbing wavelengths $\lambda>1.4 \mu \mathrm{m}$ ) but slightly smaller than those for distorted Koch fractals. The implications of these differences for snow albedo are considered in Sect. 7.

While the co-albedo values in Fig. 6b are strongly wavelength dependent through $m_{\mathrm{i}}$, the effects of shape on absorption can be distinguished more clearly by considering the non-dimensional absorption parameter (Kokhanovsky and Zege, 2004; Kokhanovsky, 2013)

$\xi=\frac{C_{\mathrm{abs}}}{\gamma V}=\frac{Q_{\mathrm{ext}} P \beta}{\gamma V}$,

where $C_{\text {abs }}$ is the absorption cross section, $Q_{\text {ext }}$ the extinction efficiency, $P$ the projected area and $V$ the particle volume, and $\gamma=4 \pi m_{\mathrm{i}} / \lambda$, where $m_{\mathrm{i}}$ is the imaginary part of ice refractive index. Figure $6 \mathrm{c}$ displays $\xi$ at the wavelengths $\lambda=0.199-1.4 \mu \mathrm{m}$, where absorption by snow is relatively weak. Consistent with the co-albedo values (Fig. 6b) and previous studies (e.g. Kokhanovsky and Nauss, 2005), Fig. 6c indicates that absorption is generally stronger for non- 

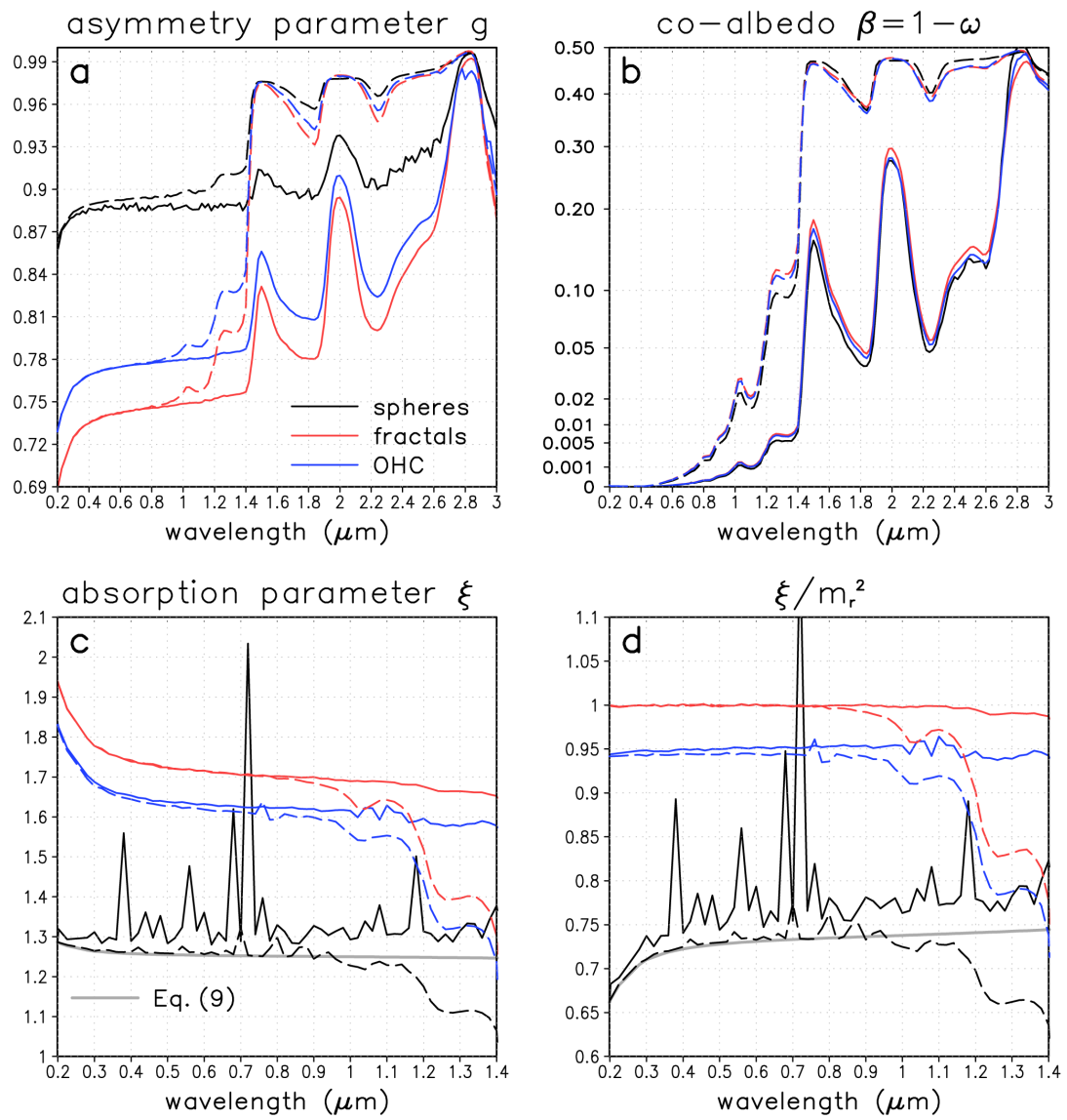

Figure 6. Comparison of single-scattering properties for spheres (black lines), distorted Koch fractals with $t=0.18$ (red) and the optimized habit combination (blue), for $r_{\mathrm{vp}}=50 \mu \mathrm{m}$ (solid lines) and $r_{\mathrm{vp}}=1000 \mu \mathrm{m}$ (dashed lines), for a monodisperse size distribution. (a) Asymmetry parameter $g$; (b) single-scattering co-albedo $\beta=1-\omega$; (c) non-dimensional absorption parameter $\xi$ (Eq. 8); and (d) $\xi$ divided by the real part of refractive index squared. In (c and $\mathbf{d})$, the grey line represents Eq. (9).

spherical than spherical particles for the same $r_{\mathrm{vp}}$. The difference is particularly clear in the visible region, where $\xi \leq 1.3$ for spheres (except for some spikes that occur in the Mie solution especially for $r_{\mathrm{vp}}=50 \mu \mathrm{m}$ ), $\approx 1.7$ for the Koch fractals and slightly over 1.6 for the $\mathrm{OHC}$.

At wavelengths beyond $\lambda=1.0 \mu \mathrm{m}, \xi$ tends to decrease especially for the larger particle size $r_{\mathrm{vp}}=1000 \mu \mathrm{m}$ considered, as absorption no longer increases linearly with $m_{\mathrm{i}}$. Furthermore, in the UV region, Koch fractals and the OHC show a distinct increase in $\xi$ with decreasing wavelength. This is related to the corresponding increase of the real part of the refractive index $m_{\mathrm{r}}$. Interestingly, it is found that for these shape assumptions, absorption scales linearly with $m_{\mathrm{r}}^{2}$; furthermore, for Koch fractals $\xi / m_{\mathrm{r}}^{2} \approx 1$ when absorption is weak (Fig. 6d). For spheres, the dependence of $\xi$ on $m_{\mathrm{r}}$ is weaker. Equation (4) in Bohren and Nevitt (1983) provides the absorption efficiency of weakly absorbing spheres in the limit of geometric optics, which can be rewritten in terms of $\xi$ as $\xi=\frac{m_{\mathrm{r}}^{3}-\left(m_{\mathrm{r}}^{2}-1\right)^{3 / 2}}{m_{\mathrm{r}}}=m_{\mathrm{r}}^{2}-\frac{\left(m_{\mathrm{r}}^{2}-1\right)^{3 / 2}}{m_{\mathrm{r}}}$.

For $r_{\mathrm{vp}}=1000 \mu \mathrm{m}, \xi$ for spheres follows this approximation closely until $\lambda \approx 1.0 \mu \mathrm{m}$ (Fig. $6 \mathrm{c}$ and d). However, it appears that for Koch fractals, only the first term should be included.

It should be noted that $\xi$ for the $\mathrm{OHC}$ is not independent of that for Koch fractals (due to the scaling of co-albedo in Eqs. (6) and (7)). However, we found that $\xi$ also scales linearly with $m_{\mathrm{r}}^{2}$ for Gaussian spheres (this was tested for $\sigma=0.17, v=2.9, n_{\max }=15$ ), suggesting that this might apply more generally to complex non-spherical particles.

Finally, it should be recalled that our choice of the OHC was based on phase function observations at the wavelength $\lambda=0.80 \mu \mathrm{m}$. At this wavelength, absorption is so weak that it has very little impact on the phase function. Therefore, these observations cannot be used to constrain absorption by snow. In spite of this, we think it is worth providing a co-albedo parameterization based on the OHC (Eq. 11 in Sect. 6.2). The reason for this is that snow grains are distinctly non-spherical, and for non-spherical particles, $\xi$ 
and $\beta$ are, in general, systematically larger than those for spheres, as demonstrated by Fig. 6. In fact, considering the wavelength $\lambda=0.80 \mu \mathrm{m}$, the values of $\xi$ integrated over the size distribution defined by Eq. (3) are, for the large majority of the non-spherical shapes considered, between 1.55 and 1.75 , the value for the $\mathrm{OHC}$ being $\xi=1.62$ (Table 1). The corresponding value for spheres is substantially lower: $\xi=1.29$. Thus, while we cannot constrain $\xi$ or $\beta$ precisely, it is very likely that the actual values for snow exceed those for spheres.

\section{Parameterizations for the single-scattering properties of snow}

In this section, parameterization equations are provided for the computation of snow SSPs (extinction efficiency $Q_{\text {ext }}$, single-scattering co-albedo $\beta$, asymmetry parameter $g$ and scattering phase function $P_{11}\left(\theta_{\mathrm{S}}\right)$ ) for the $\mathrm{OHC}$ discussed above. The parameterizations are provided for the size range $r_{\mathrm{vp}}=10-2000 \mu \mathrm{m}$ and wavelength range $\lambda=0.199-2.70 \mu \mathrm{m}$. They are expressed in terms of the size parameter $x$ and real and imaginary parts of refractive index $\left(m_{\mathrm{r}}\right.$ and $\left.m_{\mathrm{i}}\right)$. Here, the size parameter defined with respect to the volume-toprojected area equivalent radius is used:

$x=x_{\mathrm{vp}}=2 \pi \frac{r_{\mathrm{vp}}}{\lambda}$.

For the OHC, the size parameter defined with respect to the projected area is $x_{\mathrm{p}} \approx 1.535 x_{\mathrm{vp}}$.

\subsection{Extinction efficiency}

The extinction efficiency $Q_{\text {ext }}$ for the $\mathrm{OHC}$ is displayed in Fig. 7. For most of the wavelength and size region considered, $Q_{\text {ext }}$ is within $1 \%$ of the asymptotic value $Q_{\text {ext }}=2$ for particles that are large compared to the wavelength. Note that the deviations from $Q_{\text {ext }}=2$ are probably somewhat underestimated because the $\mathrm{OHC}$ includes Koch fractals, for which $Q_{\text {ext }} \equiv 2$ due to the use of geometric optics. For simplicity, we assume this value in our parameterization, while acknowledging that the actual value tends to be slightly higher especially for small snow grains in the near-IR region.

\subsection{Single-scattering co-albedo}

The single-scattering co-albedo is parameterized as

$\beta=0.470\left\{1-\exp \left[-2.69 x_{\mathrm{abs}}\left(1-0.31 \min \left(x_{\mathrm{abs}}, 2\right)^{0.67}\right)\right]\right\}$,

where the size parameter for absorption is defined as

$x_{\mathrm{abs}}=\frac{2 \pi r_{\mathrm{vp}}}{\lambda} m_{\mathrm{i}} m_{\mathrm{r}}^{2}$.

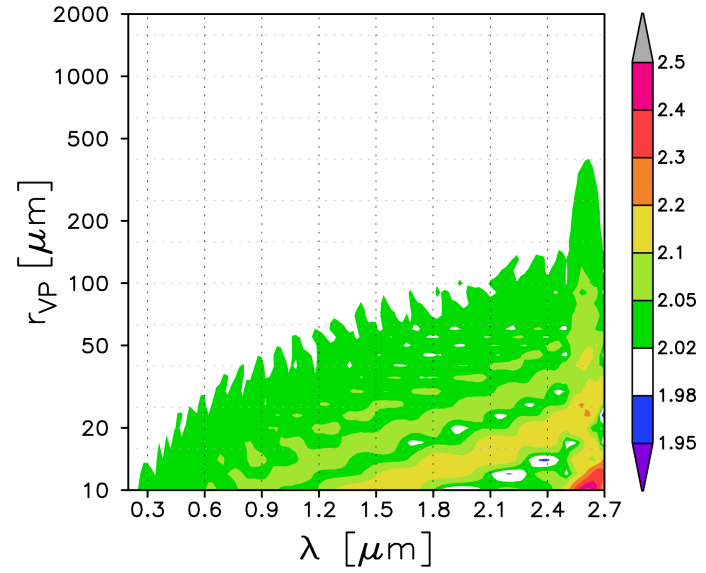

Figure 7. Extinction efficiency $Q_{\text {ext }}$ for the optimized habit combination as a function of wavelength $(\lambda)$ and volume-to-projected area equivalent radius $\left(r_{\mathrm{vp}}\right)$.

The general form of this parameterization was inspired by the ice crystal optics parameterization of van Diedenhoven et al. (2014); however, our definition of $x_{\text {abs }}$ differs from theirs in that the factor $m_{\mathrm{r}}^{2}$ is included based on the findings of Fig. $6 c$ and d. The performance of this parameterization is evaluated in Fig. 8a and c. In Fig. 8a, the parameterized values (shown with contours) follow extremely well the reference values computed for the OHC (shading). The relative errors $\Delta \beta / \beta$ are mostly below $1 \%$; errors larger than $3 \%$ (and locally even $>10 \%$ ) occur only for small snow grains $\left(r_{\mathrm{vp}}<50 \mu \mathrm{m}\right)$ at wavelengths $\lambda>1.2 \mu \mathrm{m}$. The rms value of the relative errors (computed over 125 values of $\lambda \in[0.199,2.7 \mu \mathrm{m}]$ and 48 roughly logarithmically spaced values of $\left.r_{\mathrm{vp}} \in[10,2000 \mu \mathrm{m}]\right)$ is $1.4 \%$.

\subsection{Asymmetry parameter}

The asymmetry parameter is parameterized as

$g=1-1.146\left[m_{\mathrm{r}}-1\right]^{0.8}[0.52-\beta]^{1.05}\left[1+8 x_{\mathrm{vp}}^{-1.5}\right]$,

where the parameter values were determined by trial and error, with the aim of minimizing the rms error in $g$. The form of this parameterization reflects how $g$ decreases with increasing $m_{\mathrm{r}}$, increases with increasing absorption (i.e. increasing co-albedo $\beta$ ) and increases slightly with increasing size parameter $x_{\mathrm{vp}}$ even at non-absorbing wavelengths, in part because the diffraction peak becomes narrower. In practice, the co-albedo $\beta$ plays the most important role (cf. van Diedenhoven et al., 2014), which explains the general increase of $g$ with increasing $r_{\mathrm{vp}}$ in the near-IR region (Fig. 8b). The parameterized values of $g$ (shown with contours in Fig. 8b) follow the reference values (shading) very well. Note that when producing these results, parameterized rather than exact $\beta$ was used in Eq. (13). The differences from the reference are mostly below 0.001 at the weakly ab- 

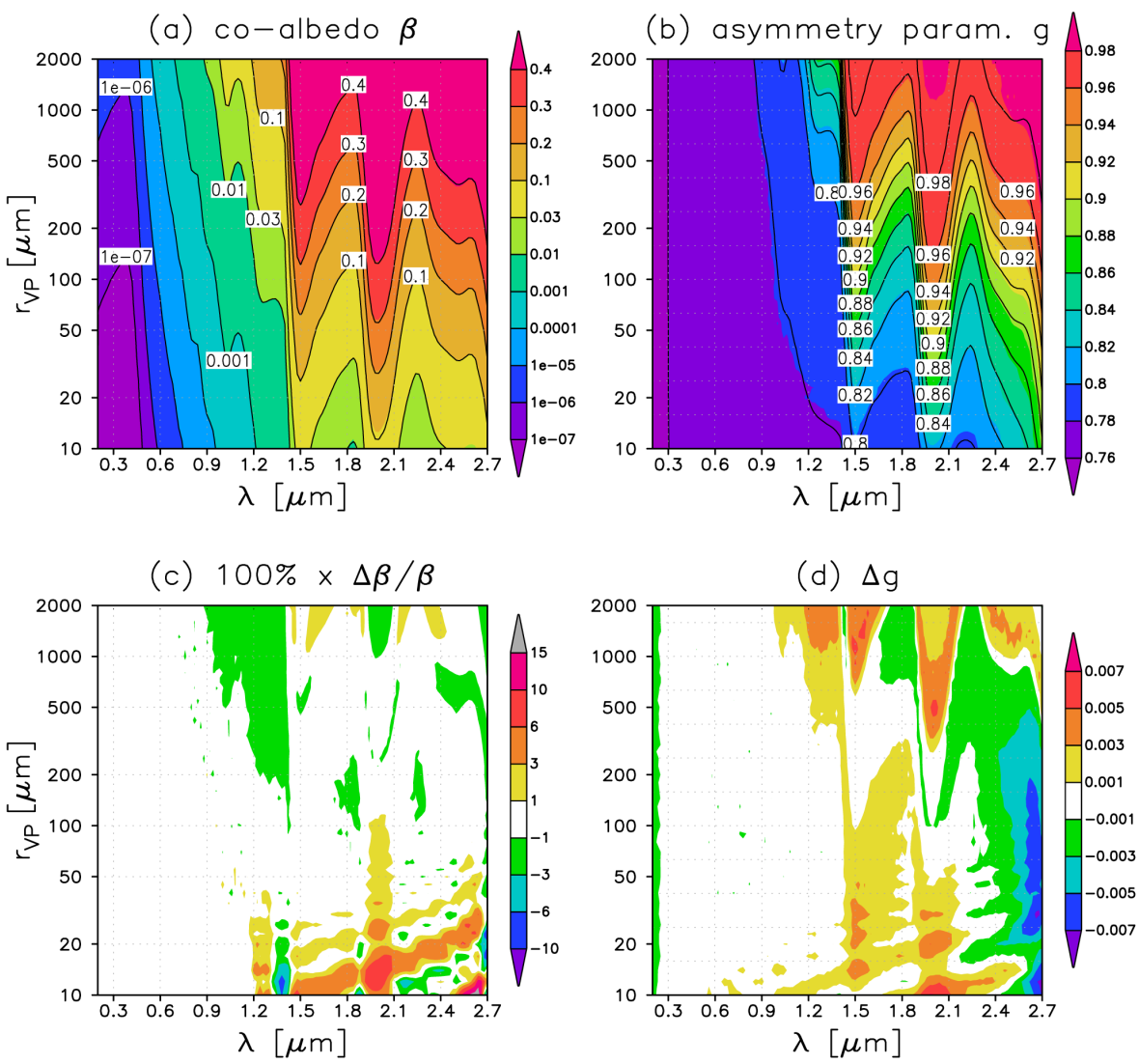

Figure 8. Comparison of (a) parameterized single-scattering co-albedo $\beta$ (contours) with the reference values computed for the OHC (shading) and (b) parameterized asymmetry parameter $g$ (contours) with the reference values (shading). (c) Relative errors (\%) in the parameterized co-albedo. (d) Absolute errors in the parameterized asymmetry parameter.

sorbing wavelengths up to $\lambda=1.4 \mu \mathrm{m}$, and while larger differences up to $|g|=0.007$ occur at the strongly absorbing wavelengths (Fig. 8d), the overall rms error is only 0.0019 .

\subsection{Phase function}

The phase function parameterization consists of three terms,

$P_{11}\left(\theta_{\mathrm{s}}\right)=w_{\text {diff }} P_{\text {diff }}\left(\theta_{\mathrm{s}}\right)+w_{\text {ray }} P_{\text {ray }}\left(\theta_{\mathrm{s}}\right)+P_{\text {resid }}\left(\theta_{\mathrm{s}}\right)$,

which represent contributions due to diffraction, due to the ray tracing part and a residual that corrects for errors made in approximating the former two parts. The weight factors for diffraction $w_{\text {diff }}$ and ray tracing $w_{\text {ray }}$ are given by

$w_{\text {diff }}=\frac{1}{Q_{\text {ext }} \omega} \approx \frac{1}{2 \omega}$,

$w_{\text {ray }}=\frac{Q_{\text {ext }} \omega-1}{Q_{\text {ext }} \omega} \approx \frac{2 \omega-1}{2 \omega}$,

where the latter form assumes $Q_{\text {ext }}=2$ (e.g. Macke et al., 1996).

It should be noted that in practice, the division of the phase function expressed by Eq. (14) is conceptual rather than rigorous. The fitting was based on the total phase function rather than the diffraction and ray tracing parts separately, as these two parts are not separated in the Yang et al. (2013) database. The general aim of the fitting was to minimize the rms errors in $\ln P_{11}$.

For diffraction, the HG phase function (Henyey and Greenstein, 1941) is used:

$P_{\text {diff }}\left(\theta_{\mathrm{s}}\right)=P_{\mathrm{HG}}\left(g_{\text {diff }}, \theta_{\mathrm{s}}\right)$.

The HG phase function is given by

$P_{\mathrm{HG}}\left(g, \theta_{\mathrm{S}}\right)=\frac{1-g^{2}}{\left[1+g^{2}-2 g \cos \theta_{\mathrm{s}}\right]^{3 / 2}}$,

and the asymmetry parameter $g_{\text {diff }}$ is approximated as

$g_{\text {diff }}=1-0.60 / x_{\mathrm{vp}}=1-0.921 / x_{\mathrm{p}}$,

where we have utilized the relation $x_{\mathrm{p}} \approx 1.535 x_{\mathrm{vp}}$ specific to the OHC. Compared to the parameterization derived by van Diedenhoven et al. (2014), Eq. (19) yields somewhat lower values of $g_{\text {diff, }}$ which to some extent compensates for the fact that the actual shape of the diffraction peak deviates from the HG phase function. Overall, this treatment of diffraction is 
a rough approximation and clearly not ideal for studies of very near-forward scattering, but it serves well the current purpose. On one hand, it improves the accuracy compared to the assumption of a delta spike, and on the other hand, the HG phase function has a very simple Legendre expansion

$P_{\mathrm{HG}}\left(g, \theta_{\mathrm{S}}\right)=\sum_{n=0}^{\infty}(2 n+1) g^{n} P_{n}\left(\cos \theta_{\mathrm{S}}\right)$,

where $P_{n}$ denotes the $n$th order Legendre polynomial. This facilitates greatly the use of $P_{\mathrm{HG}}$ in radiative transfer models such as DISORT (Stamnes et al., 1988).

The phase function for the ray tracing part is approximated as

$P_{\text {ray }}\left(\theta_{\mathrm{s}}\right)=w_{1} P_{\mathrm{HG}}\left(g_{1}, \theta_{\mathrm{s}}\right)+\left(1-w_{1}\right)$,

where the latter term $1-w_{1}$ is intended to emulate the nearly flat behaviour of $P_{11}$ in the near-backward scattering directions. The weight factor for the HG part is parameterized as

$w_{1}=1-1.53 \cdot \max \left(0.77-g_{\text {ray }}, 0\right)^{1.2}$,

where $g_{\text {ray }}$ is the asymmetry parameter for the ray tracing (i.e. non-diffraction) part. It is derived from the condition $g=w_{\text {diff }} g_{\text {diff }}+w_{\text {ray }} g_{\text {ray }}$, which yields

$g_{\text {ray }}=\frac{g-w_{\text {diff }} g_{\text {diff }}}{w_{\text {ray }}}$.

The total asymmetry parameter $g$ is computed using Eq. (13) above. Finally, the asymmetry parameter $g_{1}$ needed in Eq. (21) is

$g_{1}=g_{\text {ray }} / w_{1}$.

While the sum of the first two terms of Eq. (14) already provides a reasonably good approximation of the phase function (see below), the fit can be further improved by introducing the residual $P_{\text {resid }}$, which is represented as a Legendre series. It turns out that, except for cases with strong absorption, a series including terms only up to $n=6$ yields very good results

$P_{\text {resid }}\left(\theta_{\mathrm{s}}\right)=\sum_{n=0}^{6}(2 n+1) a_{n} P_{n}\left(\cos \theta_{\mathrm{s}}\right)$,

provided that $\delta$-M scaling (Wiscombe, 1977) is applied, with a truncated fraction $f=a_{6}$. Thus,

$$
\begin{aligned}
& P_{\text {resid }}\left(\theta_{\mathrm{s}}\right) \approx P_{\text {resid }}^{*}\left(\theta_{\mathrm{s}}\right) \\
& =2 f \delta\left(1-\cos \theta_{\mathrm{s}}\right)+(1-f) \sum_{n=0}^{5}(2 n+1) \frac{a_{n}-f}{1-f} P_{n}\left(\cos \theta_{\mathrm{s}}\right) \\
& =2 a_{6} \delta\left(1-\cos \theta_{\mathrm{s}}\right)+\sum_{n=0}^{5}(2 n+1)\left(a_{n}-a_{6}\right) P_{n}\left(\cos \theta_{\mathrm{s}}\right)
\end{aligned}
$$

where $\delta$ is Dirac's delta function. What remains to be parameterized, then, are the coefficients $a_{0} \ldots a_{6}$. A rough but useful approximation is to express them as a simple function of the co-albedo $\beta$ and the asymmetry parameter $g$ :

$a_{n}=c_{1 n}+c_{2 n} \beta+c_{3 n} g+c_{4 n} \beta g$.

The parameterization coefficients $c_{m n}$ were determined by minimizing the rms errors of $a_{n}$ with the LAPACK subroutine DGELS, and they are given in Table 2 . Note specifically that the coefficients $c_{m 0}$ and $c_{m 1}$ are all 0 . The formulation of $P_{\text {diff }}$ and $P_{\text {ray }}$ ensures that the phase function (Eq. 14) is correctly normalized and that its asymmetry parameter is consistent with Eq. (13) even without considering $P_{\text {resid }}$; therefore $a_{0}=a_{1}=0$. Equivalently, the Legendre expansion may be replaced by an ordinary polynomial. This yields

$$
\begin{gathered}
P_{\text {resid }}\left(\theta_{\mathrm{s}}\right) \approx P_{\text {resid }}^{*}\left(\theta_{\mathrm{s}}\right)=2 a_{6} \delta\left(1-\cos \theta_{\mathrm{s}}\right) \\
+\sum_{n=0}^{5} b_{n}\left(\cos \theta_{\mathrm{s}}\right)^{n},
\end{gathered}
$$

where

$b_{n}=d_{1 n}+d_{2 n} \beta+d_{3 n} g+d_{4 n} \beta g$.

Here, the coefficients $d_{m n}$ were obtained directly based on the coefficients $c_{m n}$ in Eq. (27) by writing out the Legendre polynomials in Eq. (26). Their numerical values are given in Table 3. In summary, the phase function parameterization reads

$$
\begin{aligned}
P_{11}\left(\theta_{\mathrm{s}}\right) & =w_{\mathrm{diff}} P_{\mathrm{HG}}\left(g_{\mathrm{diff}}, \theta_{\mathrm{s}}\right)+w_{\text {ray }} w_{1} P_{\mathrm{HG}}\left(g_{1}, \theta_{\mathrm{s}}\right) \\
& +w_{\text {ray }}\left(1-w_{1}\right)+P_{\text {resid }}\left(\theta_{\mathrm{s}}\right),
\end{aligned}
$$

where $P_{\text {resid }}\left(\theta_{\mathrm{s}}\right)$ is given by Eq. (26) or, equivalently, by Eq. (28).

Finally, it is worth noting how this parameterization can be used in DISORT, when applying a " $\delta$-NSTR-stream" approximation for radiative transfer, NSTR being the number of streams. In this case, DISORT assumes by default a truncation factor $f=a_{\mathrm{NSTR}}$. If NSTR $>6$, the Legendre expansion for $P_{\text {resid }}$ in Eq. (26) should be formally extended to $n=$ NSTR, with $a_{n}=a_{6}$ for $n=7 \ldots$ NSTR. Thus the Legendre coefficients input to DISORT become

$p_{n}=\left\{\begin{array}{ll}1, & \text { for } n=0 \\ w_{\text {diff }} g_{\text {diff }}^{n}+w_{\text {ray }} w_{1} g_{1}^{n}+a_{n}, & \text { for } 1 \leq n \leq 6 \\ w_{\text {diff }} g_{\text {diff }}^{n}+w_{\text {ray }} w_{1} g_{1}^{n}+a_{6}, & \text { for } 7 \leq n \leq \mathrm{NSTR}\end{array}\right.$,

where we have utilized the Legendre expansion of the HG phase function in Eq. (20).

To provide a compact view of how the phase function parameterization performs, we define, analogously to Eq. (4), a cost function as the rms error of the natural logarithm of the phase function,

$\operatorname{cost}=\sqrt{\frac{\int_{0^{\circ}}^{180^{\circ}}\left(\ln P_{11}^{\mathrm{param}}-\ln P_{11}^{\mathrm{OHC}}\right)^{2} \sin \theta_{\mathrm{s}} \mathrm{d} \theta_{\mathrm{s}}}{\int_{0^{\circ}}^{180^{\circ}} \sin \theta_{\mathrm{s}} \mathrm{d} \theta_{\mathrm{s}}}}$, 

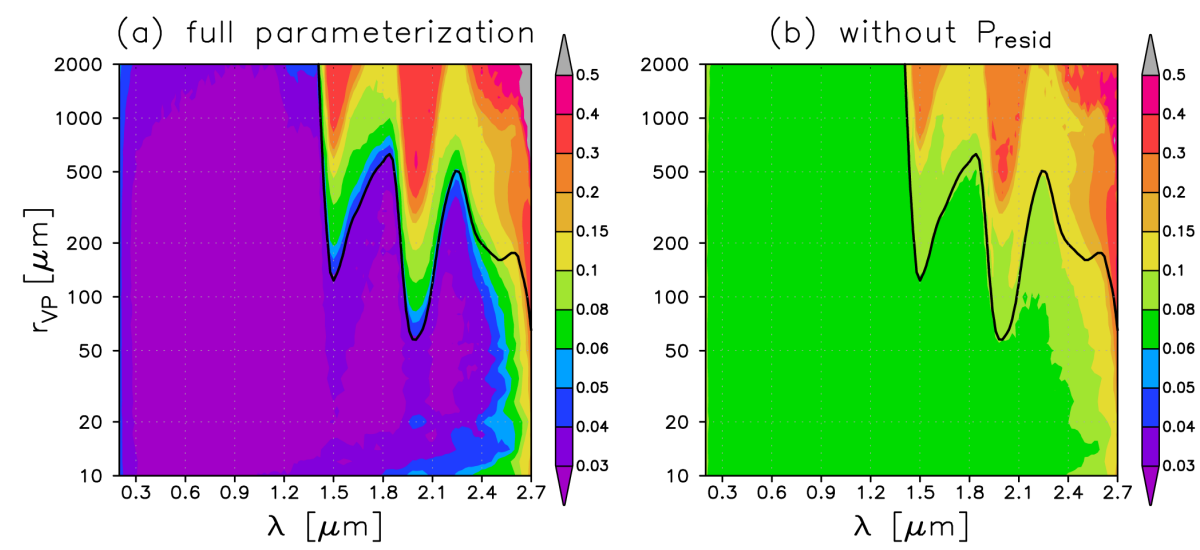

Figure 9. Cost function for the phase function parameterization as defined by Eq. (32) for (a) the full parameterization (Eq. 14) and (b) without the term $P_{\text {resid }}$. The black solid line indicates, for reference, a co-albedo value of $\beta=0.3$, which approximately corresponds to a spherical albedo of 0.03 for an optically thick snow layer.

Table 2. Parameterization coefficients appearing in Eq. (27).

\begin{tabular}{rrrrr}
\hline & $c_{1 n}$ & $c_{2 n}$ & $c_{3 n}$ & $c_{4 n}$ \\
\hline$n=0$ & 0.00000 & 0.00000 & 0.00000 & 0.00000 \\
$n=1$ & 0.00000 & 0.00000 & 0.00000 & 0.00000 \\
$n=2$ & -0.01400 & -0.10367 & 0.02144 & 0.08903 \\
$n=3$ & -0.13184 & -0.01741 & 0.16890 & -0.06365 \\
$n=4$ & -0.20878 & -0.03438 & 0.27353 & -0.10418 \\
$n=5$ & -0.29763 & -0.06931 & 0.38501 & -0.11329 \\
$n=6$ & -0.32153 & -0.10691 & 0.41282 & -0.07934 \\
\hline
\end{tabular}

where $P_{11}^{\text {param }}$ is the parameterized phase function and $P_{11}^{\mathrm{OHC}}$ is the reference value, defined here as the "exact" phase function computed for the OHC. Figure 9a shows the cost function for the full phase function parameterization, and Fig. 9b shows that for a simpler parameterization that includes only the first two terms of Eq. (14) (i.e. $P_{\text {resid }}$ is excluded). Note that the parameterized phase function is computed here using parameterized (rather than exact) values of $Q_{\text {ext }}, \beta$ and $g$.

Most importantly, Fig. 9a shows that in a large part of the wavelength and size domain, the accuracy of the full parameterization is very high, with cost function values $\leq 0.03$. This corresponds to a typical relative accuracy of $3 \%$ in the computed phase function, as compared with the reference values for the OHC. The primary exception is that substantially larger errors occur for large snow grains at the strongly absorbing wavelengths in the near-IR region. In broad terms, the accuracy starts to degrade appreciably when $\beta>0.3$, that is, in cases in which snow reflectance is quite low $(\beta=0.3$ corresponds roughly to a spherical albedo of 0.03 for an optically thick snow layer). At the largest wavelengths considered $(\lambda>2.5 \mu \mathrm{m})$, somewhat larger values of the cost function also occur for smaller values of $r_{\mathrm{vp}}$ and $\beta$. The cost function for the simplified parameterization (Fig. 9b) shows mainly the same qualitative features as the full parameter-
Table 3. Parameterization coefficients appearing in Eq. (29).

\begin{tabular}{rrrrr}
\hline & $d_{1 n}$ & $d_{2 n}$ & $d_{3 n}$ & $d_{4 n}$ \\
\hline$n=0$ & -0.06679 & 0.34357 & 0.09553 & -0.42542 \\
$n=1$ & -0.53413 & 0.15642 & 0.74905 & -0.62700 \\
$n=2$ & -1.49866 & -2.42334 & 1.76580 & 2.10118 \\
$n=3$ & 1.01884 & -2.05239 & -1.59160 & 3.54237 \\
$n=4$ & 4.43936 & 2.85558 & -5.48475 & -0.97817 \\
$n=5$ & 2.07065 & 3.25673 & -2.40933 & -2.94094 \\
\hline
\end{tabular}

ization in Fig. 9a; however, the cost function values in the weakly absorbing cases are $\approx 0.07$, in contrast to the values of $\approx 0.03$ for the full parameterization.

Figure 10 displays examples of phase function for nine combinations of $\lambda$ and $r_{\mathrm{vp}}$. In the weakly absorbing cases in Fig. 10a-c, and also at the more strongly absorbing wavelength $\lambda=1.50 \mu \mathrm{m}$ for $r_{\mathrm{vp}}=10 \mu \mathrm{m}$ and $r_{\mathrm{vp}}=100 \mu \mathrm{m}$ (Fig. 10d and e), the full parameterization follows extremely well the reference phase function computed for the $\mathrm{OHC}$, to the extent that the curves are almost indistinguishable from each other. Even at $\lambda=2.00 \mu \mathrm{m}$, the deviations from the reference are generally small in the cases with relatively small snow grains $\left(r_{\mathrm{vp}}=10 \mu \mathrm{m}\right.$ and $r_{\mathrm{vp}}=100 \mu \mathrm{m}$; Fig. $10 \mathrm{~g}$ and $\left.\mathrm{h}\right)$, although backward scattering is slightly overestimated in the latter case. In contrast, in cases with very strong absorption and large snow grains $\left(r_{\mathrm{vp}}=1000 \mu \mathrm{m}\right.$ for $\lambda=1.50 \mu \mathrm{m}$ and $\lambda=2.00 \mu \mathrm{m}$ in Fig. 10f and i) there are more substantial deviations from the reference. Here, the parameterized phase function is generally underestimated in the backscattering hemisphere and overestimated at $\theta_{\mathrm{s}}<30^{\circ}$ especially for $\lambda=2.00 \mu \mathrm{m}, r_{\mathrm{vp}}=1000 \mu \mathrm{m}$. Furthermore, the Legendre expansion in $P_{\text {resid }}$ leads to oscillations in the backscattering hemisphere which do not occur in the reference phase function. Again, it should be noted that the largest errors occur 

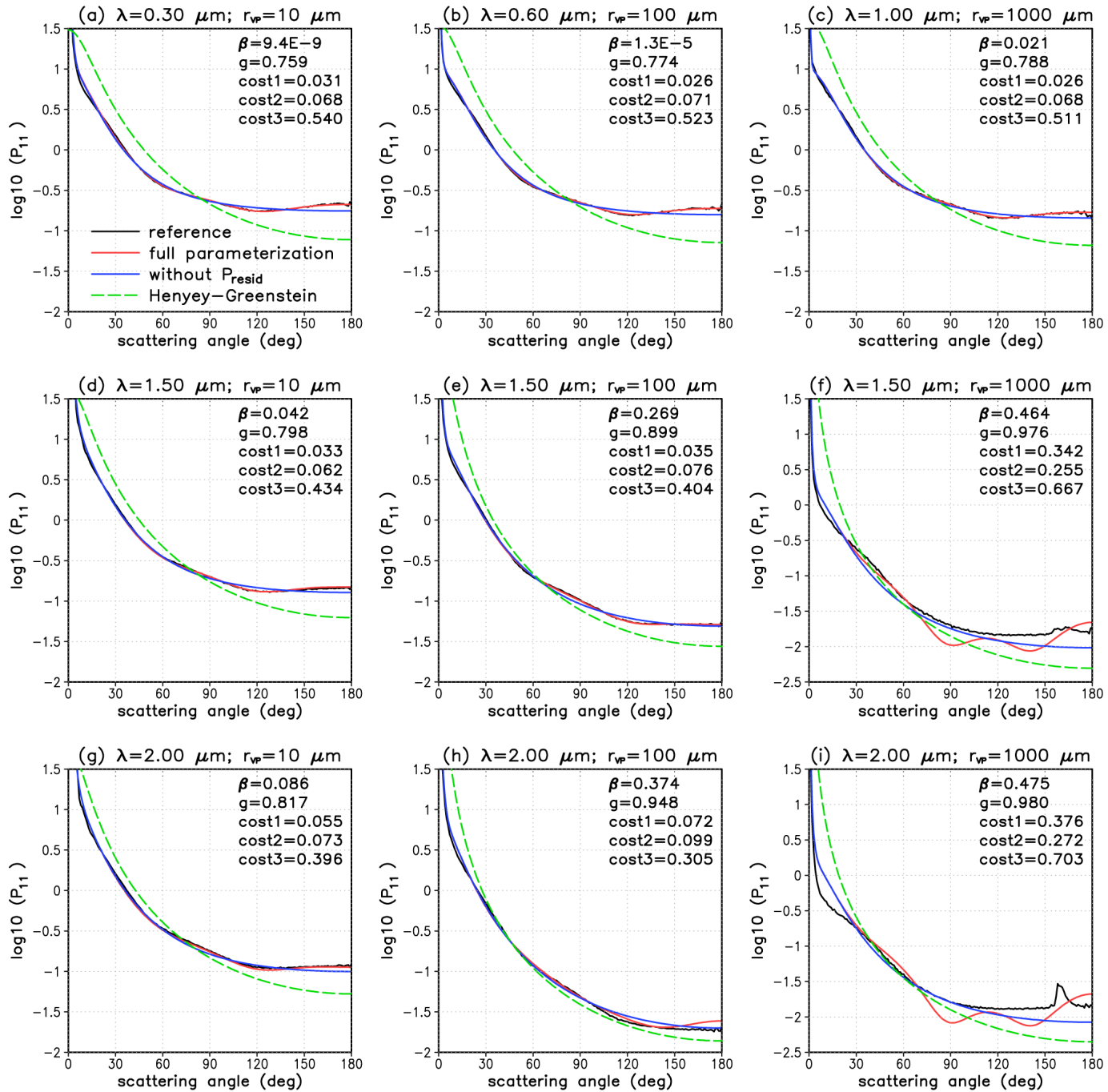

Figure 10. Examples of the reference phase function computed for the OHC (black lines) and of the parameterized phase function for the full parameterization (red lines), the simplified parameterization without the term $P_{\text {resid }}$ in Eq. (14) (blue lines) and the Henyey-Greenstein phase function with asymmetry parameter defined by Eq. (13) (dashed green lines) for nine combinations of wavelength $\lambda$ and volume-to-projected area equivalent radius $r_{\mathrm{vp}}$. For reference, the values of single-scattering co-albedo $\beta$, asymmetry parameter $g$ and cost functions for the full parameterization (cost1), for the simplified parameterization (cost2) and for the Henyey-Greenstein phase function (cost3) are listed in each panel.

in cases in which snow is very "dark": the spherical albedo corresponding to the cases in Fig. 10f and i is only $\sim 0.005$.

In many respects, the simplified parameterization (i.e. without $\left.P_{\text {resid }}\right)$ produces quite similar phase functions as the full parameterization. Two differences can be noted. First, the simplified parameterization does not capture the slight increase in phase function at angles larger than $\theta_{\mathrm{S}} \approx 120-130^{\circ}$, which is present in the reference and full parameterization phase functions and was also suggested by the CLIMSLIP data for blowing snow at $\lambda=0.80 \mu \mathrm{m}$, along with the other phase functions in Fig. 1b. Second, in the cases with very strong absorption (Fig. 10f and i) the simplified phase function avoids the oscillations seen in the full parameterization.

The utility of providing a phase function parameterization is further demonstrated by showing in Fig. 10, for comparison, the HG phase function computed using the asymmetry parameter from Eq. (13). The differences from the reference phase function are systematic. The scattering in the diffraction peak is underestimated (although this is not properly seen from Fig. 10), but otherwise forward scattering is overestimated until a scattering angle of $\approx 35-80^{\circ}$, depending on the case. Conversely, at sideward and backscattering angles, scattering is underestimated. Consequently, the cost function values for the HG phase function given in Fig. 10 

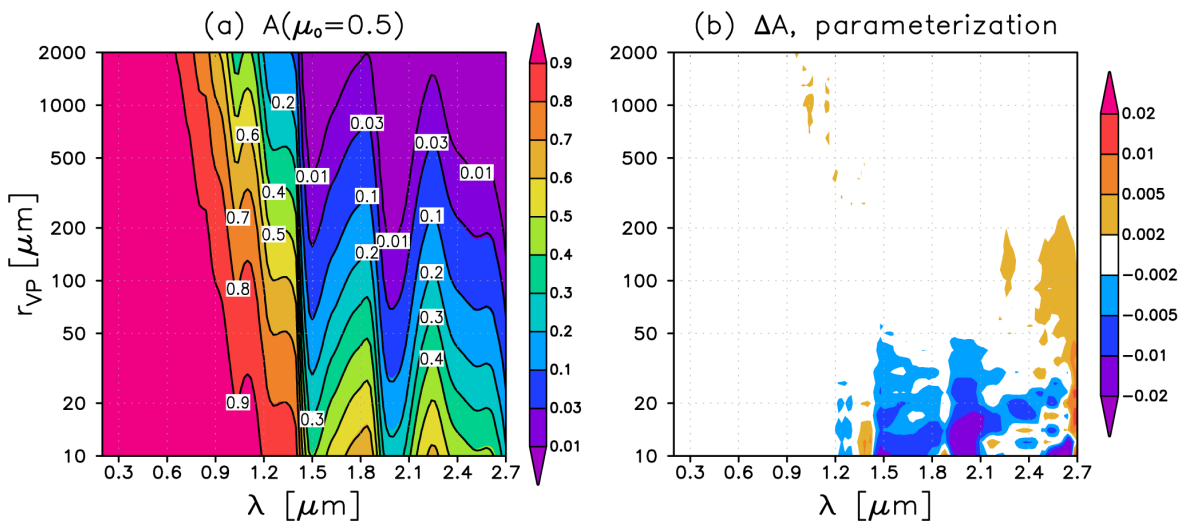

(c) $\Delta \mathrm{A}$, Koch fractals
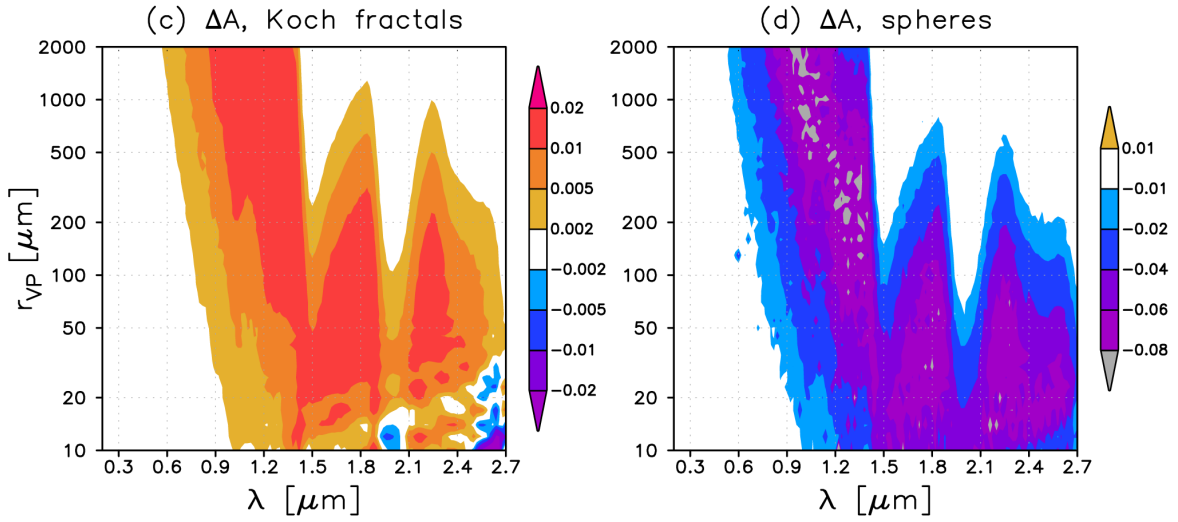

Figure 11. Albedo of a semi-infinite snow layer for direct incident radiation with the cosine of zenith angle $\mu_{0}=0.5$. (a) Reference values computed for the OHC (shading) and values for the full snow optics parameterization (contours). The difference (b) between the parameterization and the reference, $(\mathbf{c})$ between distorted Koch fractals $(t=0.18)$ and the reference and (d) between spheres and the reference. Note that the colour scale differs between the figure panels.

substantially exceed those for both the full and simplified phase function parameterizations.

\section{Radiative transfer applications}

In this section, we consider the impact of snow optics assumptions on snow spectral albedo $A$ and reflected radiances $L^{\uparrow}$. The purpose is, on one hand, to evaluate the accuracy of the proposed snow SSP parameterization and, on the other hand, to compare the results obtained with three shape assumptions: spheres, second-generation Koch fractals (distorted with $t=0.18$ ) and the OHC proposed here. Throughout this section, the results for the $\mathrm{OHC}$ are used as the reference, although it is clear that they cannot be considered an absolute benchmark for scattering by snow. The radiative transfer computations were performed with DISORT (with 32 streams, $\delta$-M scaling included), assuming an optically thick (i.e. semi-infinite) layer of pure snow with a monodisperse size distribution.

Like most other solar radiative transfer studies involving snow, close-packed effects are ignored in the calculations. It has been shown by Kokhanovsky (1998) that, at least as a first approximation, they do not have a pronounced impact on the snow reflectance.

First, snow albedo as a function of $\lambda$ and $r_{\mathrm{vp}}$ is considered in Fig. 11. Direct incident radiation with a cosine of zenith angle $\mu_{0}=\cos \theta_{0}=0.5$ is assumed. Figure 11a demonstrates the well-known features of snow albedo: the values are very high in the UV and visible region and decrease with increasing particle size in the near-IR. The results computed using the parameterized snow optical properties $Q_{\text {ext }}, \beta, g$ and $P_{11}$ are almost indistinguishable from those obtained using the "exact" optical properties for the OHC. The differences between these two are mostly within 0.002 (Fig. 11b), although larger differences up to 0.02 occur for very small snow grains $\left(r_{\mathrm{vp}} \approx 10-20 \mu \mathrm{m}\right)$ at wavelengths with strong absorption by snow $(\lambda>1.4 \mu \mathrm{m})$. These results are only weakly sensitive to the assumed direction of incident radiation. Furthermore, while the parameterized albedo values were computed using the full phase function parameterization, the values for the simplified parameterization (without $P_{\text {resid }}$ in Eq. (14)) differed very little from them, mostly by less than 0.001 .

For distorted Koch fractals, the albedo values are higher than those for the OHC, but the difference is rather small, 
at most 0.017 (Fig. 11c). Conversely, for spheres the albedo values are lower, with largest negative differences of -0.08 from the reference (Fig. 11d). This stems from the higher asymmetry parameter of spheres, which is only partly compensated by their lower co-albedo (Fig. 6). To put it another way, for a given albedo $A$ in the near-IR region, a smaller (slightly larger) particle size is required for spheres (for distorted Koch fractals) than for the OHC.

To compare the simulated radiance distributions to the reference, we next consider the root-mean-square error in the logarithm of reflected radiances integrated over the hemisphere:

\section{LOGRMSE $=$}

$$
\sqrt{\frac{1}{2 \pi} \int_{0}^{2 \pi} \int_{0}^{\pi / 2}\left[\ln L^{\uparrow}(\theta, \phi)-\ln L_{\mathrm{OHC}}^{\uparrow}(\theta, \phi)\right]^{2} \sin \theta \mathrm{d} \theta \mathrm{d} \phi,}
$$

where $\theta$ and $\phi$ denote the zenith angle and azimuth angle respectively and $L_{\mathrm{OHC}}^{\uparrow}$ is the radiance in the reference computations for the OHC. Figure 12a-c show LOGRMSE as a function of particle size and wavelength for the full parameterization for three directions of incident radiation $\left(\mu_{0}=0.8, \mu_{0}=0.4\right.$ and $\mu_{0}=0.1$, corresponding to $\theta_{0}=36.9^{\circ}, \theta_{0}=66.4^{\circ}$ and $\theta_{0}=84.3^{\circ}$ ). For weakly absorbing wavelengths up to $\lambda=1.4 \mu \mathrm{m}$, the performance of the parameterization is extremely good for all particle sizes, with values of LOGRMSE $<0.01$ for $\mu_{0}=0.8$ and $\mu_{0}=0.4$ and between 0.01 and 0.02 for $\mu_{0}=0.1$. LOGRMSE $\sim 0.01 \mathrm{im}$ plies a typical relative accuracy of $\sim 1 \%$ in the reflected radiances. The accuracy in radiances at weakly absorbing wavelengths is even higher than that in the phase function (Fig. 9a) because strong multiple scattering diminishes the effect of phase function errors. At wavelengths $\lambda>1.4 \mu \mathrm{m}$, LOGRMSE increases, not only due to larger phase function errors but also because multiple scattering is reduced due to stronger absorption. Even here, LOGRMSE stays mainly below 0.05 for relatively small snow grains $\left(r_{\mathrm{vp}}<100 \mu \mathrm{m}\right)$, but substantially larger errors occur in the cases with large and strongly absorbing grains, consistent with the modest accuracy of the phase function parameterization in these cases (Fig. 9a). These errors depend only weakly on $\mu_{0}$. It should be noted that the largest relative errors occur in cases where the reflected radiances and radiance errors are small in an absolute sense and probably matter little for practical applications.

Values of LOGRMSE obtained using the simplified phase function parameterization are shown in Fig. 12d-f. Consistent with the phase function errors (cf. Fig. 9a vs. b), the simplified parameterization is slightly less accurate in simulating reflected radiances than the full parameterization except for the most strongly absorbing cases. Nevertheless, the accuracy is quite high for the weakly absorbing cases; LOGRMSE ranging from $\sim 0.01$ (or even less) for $\mu_{0}=0.8$ to $\sim 0.03$ for $\mu_{0}=0.1$.
For comparison, Fig. $12 \mathrm{~g}$ and h show LOGRMSE computed for distorted Koch fractals and spheres (for $\mu_{0}=0.4$ only). Unsurprisingly, LOGRMSE is generally smaller for Koch fractals than for spheres (e.g. 0.05-0.10 in weakly absorbing cases compared to $\sim 0.20$ for spheres). In both cases, again excepting large particles at strongly absorbing wavelengths, the values of LOGRMSE are substantially larger than those associated with the snow SSP parameterization. This indicates that in general, numerical fitting errors in the parameterization are a minor issue in comparison with the radiance differences associated with different shape assumptions.

Examples of the angular distribution of reflected radiances are given in Figs. 13 and 14. Here, only a single particle size $r_{\mathrm{vp}}=200 \mu \mathrm{m}$ is considered, and the azimuth angle for incident radiation is $\phi_{0}=0^{\circ}$. In Fig. 13, results are shown for three zenith angles of incident radiation, corresponding to $\mu_{0}=0.8, \mu_{0}=0.4$ and $\mu_{0}=0.1$, for a single wavelength $\lambda=0.80 \mu \mathrm{m}$. In Fig. 14, three wavelengths are considered $(\lambda=0.30,1.40$ and $2.20 \mu \mathrm{m})$ but for $\mu_{0}=0.4$ only. In each figure, panels a-c display the distribution of reflected radiances in the reference calculations for the $\mathrm{OHC}$, while the remaining panels show the relative differences from the reference for distorted Koch fractals with $t=0.18$ (panels $\mathrm{d}-\mathrm{f}$ ), for spheres $(\mathrm{g}-\mathrm{i})$, for the Henyey-Greenstein phase function $(\mathrm{j}-1)$, for the full snow SSP parameterization $(\mathrm{m}-\mathrm{o})$ and for the simpler parameterization without $P_{\text {resid }}$ in Eq. (14) (p-r). For brevity, only some main points are discussed.

First, it is seen, consistent with Fig. 12, that in general the radiance distribution for spheres differs more from the reference than the distribution for Koch fractals does. For example, for $\lambda=0.80 \mu \mathrm{m}$ and $\mu_{0}=0.4$ both positive and negative differences larger than $50 \%$ occur for spheres (Fig. 13h), while for Koch fractals the differences exceed $10 \%$ only locally (Fig. 13e). Furthermore, in the same case, the radiance errors are $<1 \%$ almost throughout the $(\theta, \phi)$ domain for the full parameterization (Fig. 13n) and mostly $<2 \%$ even for the simplified parameterization (Fig. 13q). In contrast, when the HG phase function is employed in the calculations, the differences from the reference reach locally 30 and $-40 \%$ (Fig. 13k).

Second, while the results noted above for $\lambda=0.80 \mu \mathrm{m}$ and $\mu_{0}=0.4$ are also mostly valid for $\mu_{0}=0.8$ and $\mu_{0}=0.1$ and for $\lambda=0.30,1.40$ and $2.20 \mu \mathrm{m}$, some quantitative differences can be noted. When $\mu_{0}$ decreases from 0.8 to 0.1 , the pattern of reflected radiances becomes increasingly non-uniform and more sensitive to both the assumed particle shape and the errors in phase function parameterization. This occurs because the relative role of first-order scattering increases (e.g. Mischenko et al., 1999). For the same reason, the sensitivity of the radiance pattern to the phase function increases with increasing absorption. Thus, while the qualitative features are mostly similar at all wavelengths considered here, the relative differences are generally larger at $\lambda=1.40 \mu \mathrm{m}$ and $\lambda=2.20 \mu \mathrm{m}$ than at $\lambda=0.30 \mu \mathrm{m}$ and $\lambda=0.80 \mu \mathrm{m}$. Espe- 
(a) full param., $\mu_{0}=0.8$

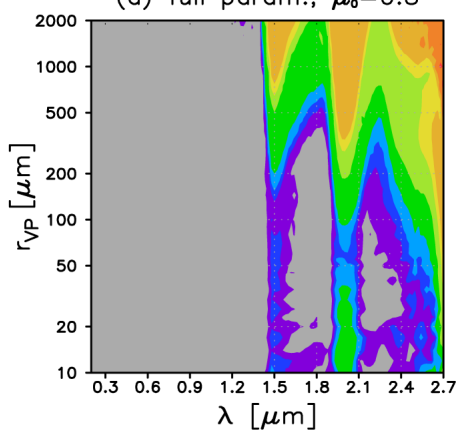

(d) without $P_{\text {resid }}, \mu_{0}=0.8$

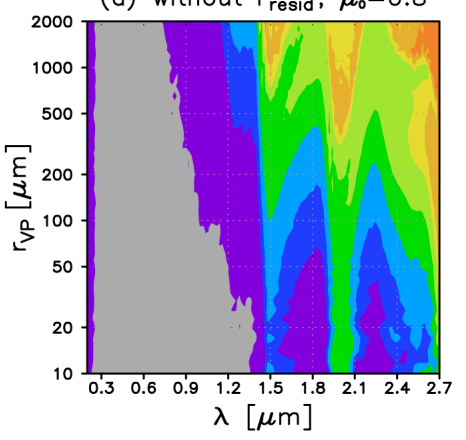

(b) full param, $\mu_{0}=0.4$

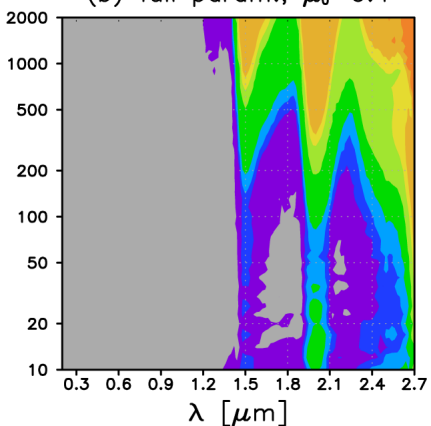

(e) without $P_{\text {resid }}, \mu_{0}=0.4$

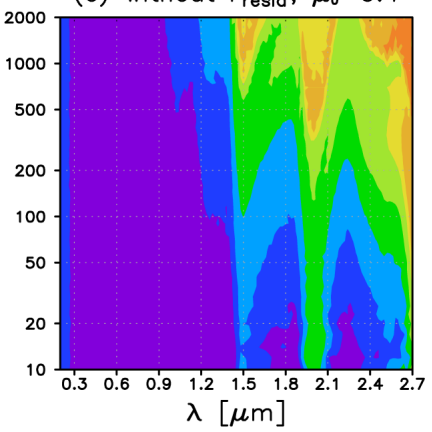

(c) full param, $\mu_{0}=0.1$

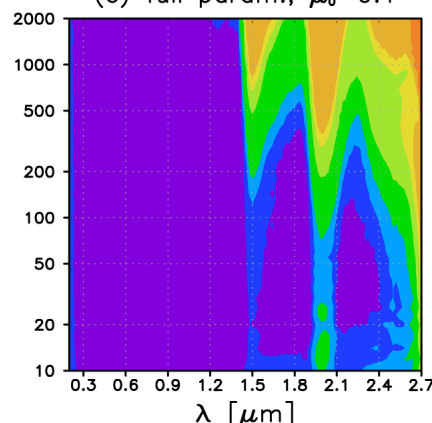

(f) without $P_{\text {resid, }} \mu_{0}=0.1$

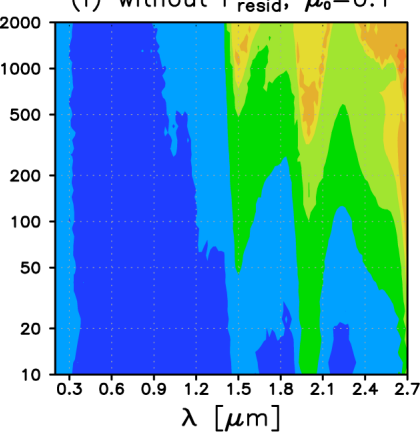

(g) Koch fractals. $\mu_{0}=0.4$

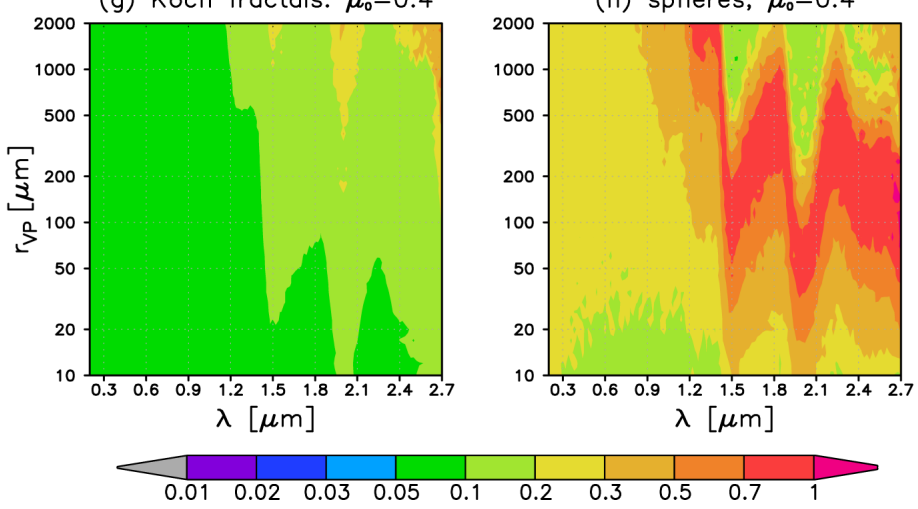

Figure 12. Root-mean-square errors in $\ln$ (radiance) (Eq. 33) for (a)-(c) the full parameterization and (d)-(f) the simpler parameterization without the term $P_{\text {resid }}$ in the phase function, as compared with reference calculations for the OHC, for three directions of incident radiation (cosine of zenith angle $\mu_{0}=0.8, \mu_{0}=0.4$ and $\mu_{0}=0.1$ ). (g) and (h) show the respective differences from the reference calculations for distorted Koch fractals $(t=0.18)$ and spheres (for $\mu_{0}=0.4$ only).

cially at the wavelength $\lambda=2.20 \mu \mathrm{m}$, at which snow absorption is quite strong and the albedo for the OHC is only 0.11 , the radiance pattern is dominated by first-order scattering and is thus very sensitive to the details of the phase function. In a relative (though not absolute) sense, the errors in parameterized radiances are also somewhat larger than at the other wavelengths considered (Fig. 14o and r).

Third, even at weakly absorbing wavelengths, the role of first-order scattering is clearly discernible: many differences in the pattern of reflected radiances can be traced directly to phase function differences. For example, considering the results for $\lambda=0.80 \mu \mathrm{m}$ for both $\mu_{0}=0.4$ and $\mu_{0}=0.1$, we note the following.

- Three regions appear in the radiance differences between distorted Koch fractals and the OHC in Fig. 13e and $\mathrm{f}$. Going from left to right, negative radiance differences occur at large values of $\theta$ and small values of $\phi$ (roughly for $\theta>65^{\circ}$ and $\phi<20^{\circ}$ ), followed by a region of positive differences and another region of negative differences (roughly for $\theta>40^{\circ}, \phi>140^{\circ}$ ). These regions occur because the phase function for Koch fractals is larger than that for the $\mathrm{OHC}$ at intermediate scattering 

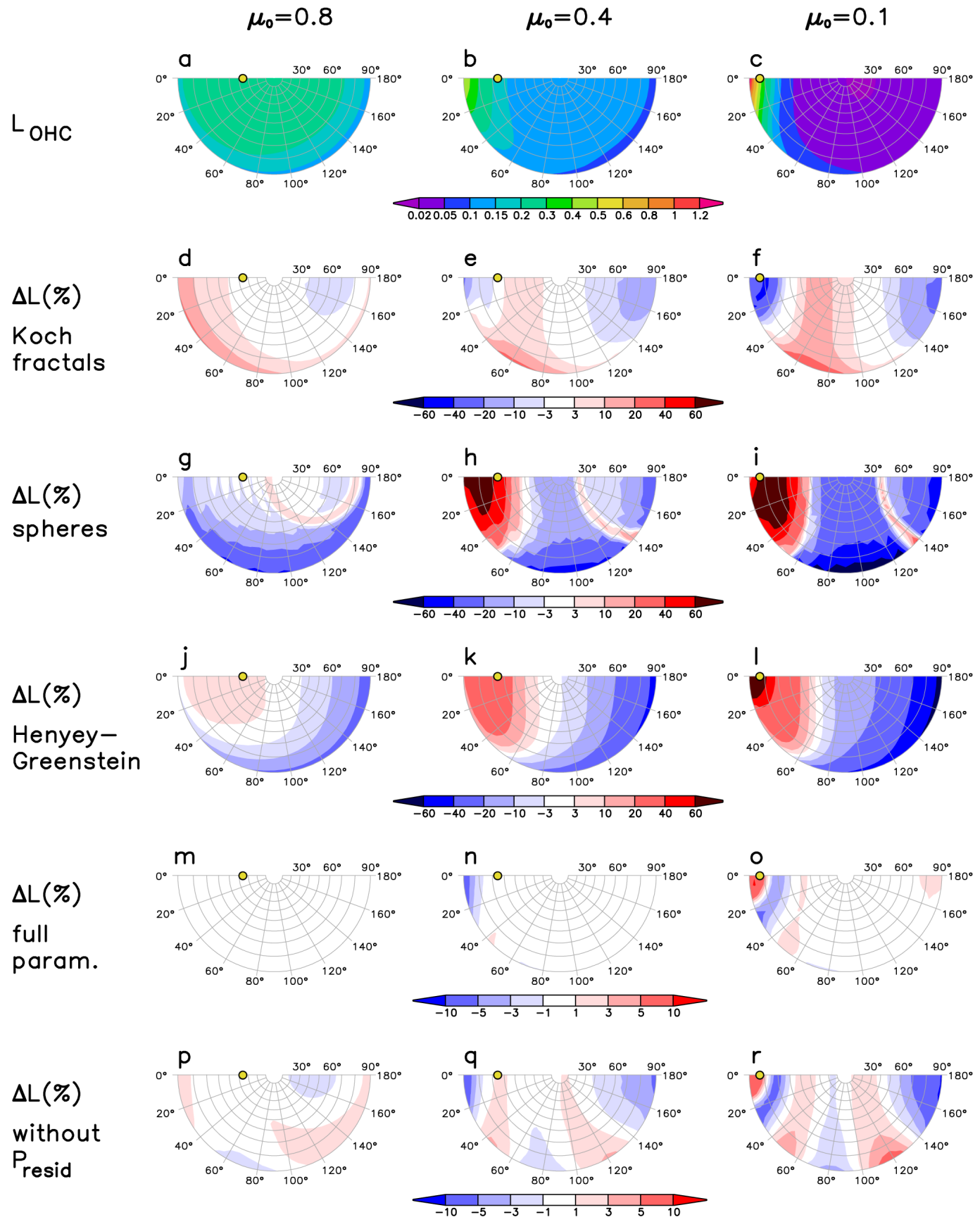

Figure 13. (a)-(c) Angular distribution of reflected radiances for the $\mathrm{OHC}$ for a single wavelength $\lambda=0.80 \mu \mathrm{m}$ and a single particle size $r_{\mathrm{vp}}=200 \mu \mathrm{m}$. The yellow sphere indicates the cosine of zenith angle for the incident radiation $\left(\mu_{0}=0.8, \mu_{0}=0.4\right.$ and $\mu_{0}=0.1$ for $(\mathbf{a})-(\mathbf{c})$ respectively). The azimuth angle for the incident radiation is $\phi_{0}=0^{\circ}$. (d)-(f) and (g)-(i) show the fractional differences in reflected radiances (in \%) from the $\mathrm{OHC}$ for distorted Koch fractals with $t=0.18$ and for ice spheres respectively. (j)-(l) show the differences from the OHC for the Henyey-Greenstein phase function (with $g$ computed using Eq. 13 and $\beta$ using Eq. 11), (m)-(o) for the full snow optics parameterization and (p)-(r) for the simpler parameterization without $P_{\text {resid }}$ in Eq. (14). Note that the colour scale in (m)-(r) differs from that in (d) $-(\mathbf{l})$.

angles $\left(29^{\circ} \leq \theta_{\mathrm{s}} \leq 134^{\circ}\right)$ but smaller in the near-forward and near-backward directions.

- For spheres in Fig. 13h and i, the reflected radiances greatly exceed those for the $\mathrm{OHC}$ for roughly $\theta>60^{\circ}$, $\phi<40^{\circ}$ because the phase function for spheres is generally larger than that for the $\mathrm{OHC}$ for $\theta_{\mathrm{s}}<54^{\circ}$. Conversely, at larger $\theta_{\mathrm{S}}$ the phase function for spheres is (mostly) considerably smaller than that for the OHC. This results in generally smaller reflected radiances for 

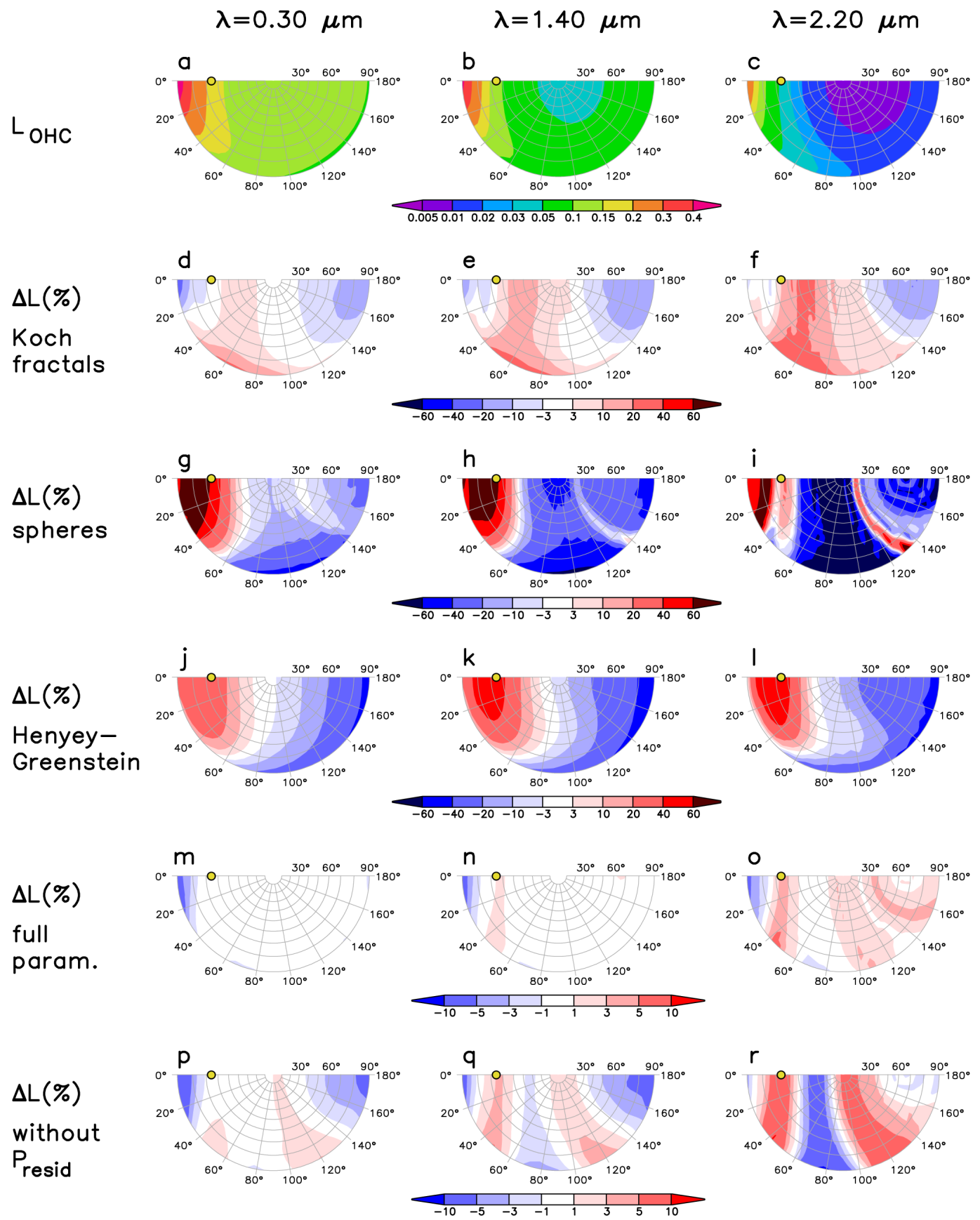

Figure 14. As Fig. 13 but for three wavelengths $\lambda=0.30,1.40$ and $2.20 \mu \mathrm{m}$ for a single value of the cosine of zenith angle for incident radiation $\mu_{0}=0.4$ and a single particle size $r_{\mathrm{vp}}=200 \mu \mathrm{m}$.

spheres in most of the $(\theta, \phi)$ domain with $\phi>50^{\circ}$. As an exception, the icebow feature for spheres at $\theta_{\mathrm{s}} \approx 135^{\circ}$ results in an arc with larger radiances for spheres than for the $\mathrm{OHC}$.

- For the HG phase function, the pattern of overestimated radiances up to $\phi \sim 60^{\circ}$ and underestimated radiances at larger azimuth angles (Fig. 13k and 1) arises because the HG phase function exceeds that for the $\mathrm{OHC}$ for $\theta_{\mathrm{s}}<80^{\circ}$ and falls below it at larger scattering angles (see also Fig. 10).

\section{Summary}

In this work, measurements of angular distribution of scattering by blowing snow made during the CLIMSLIP campaign in Svalbard were used to select a shape model for represent- 
ing the single-scattering properties of snow. An optimized habit combination consisting of SR droxtals, aggregates of SR plates and strongly distorted Koch fractals was selected. The SSPs (extinction efficiency $Q_{\text {ext }}$, single-scattering coalbedo $\beta$, asymmetry parameter $g$ and phase function $P_{11}$ ) were then computed for the $\mathrm{OHC}$ as a function of wavelength and snow grain size. Furthermore, parameterization equations were developed for the SSPs for the wavelength range $\lambda=0.199-2.7 \mu \mathrm{m}$ and for snow grain volume-to-projected area equivalent radii $r_{\mathrm{vp}}=10-2000 \mu \mathrm{m}$. The parameterizations are expressed in terms of the size parameter and real and imaginary parts of refractive index. The relative accuracy of the parameterization, as compared with the reference calculations for the $\mathrm{OHC}$, is very high for the single-scattering coalbedo and the asymmetry parameter. This is also true for the phase function parameterization in weakly and moderately absorbing cases, while in strongly absorbing cases (mainly for $\beta>0.3$ ) the accuracy deteriorates. Such strongly absorbing cases are, however, associated with small values of snow albedo and reflected radiances.

The SSPs and the resulting snow albedo and reflected radiances for the $\mathrm{OHC}$ were compared with two previously used shape assumptions for snow grains, spheres and secondgeneration Koch fractals. The asymmetry parameter for the $\mathrm{OHC}$ is distinctly smaller than that for spheres but slightly higher than that for Koch fractals. Consistent with this, snow albedo for the $\mathrm{OHC}$ is generally substantially higher (slightly lower) than that for spheres (Koch fractals) for a given snow grain size $r_{\mathrm{vp}}$. Also for the distribution of reflected radiances, spheres differ more from the OHC than Koch fractals do.

The main limitation of the current work is that the SSP parameterization is based on a rather limited observational data set. The OHC was selected using scattering measurements at a single wavelength $\lambda=0.80 \mu \mathrm{m}$ for only two cases with blowing snow. This raises several potential issues:

- The choice of the OHC based on scattering measurements only implies that it most probably does not represent properly the actual distribution of snow grain shapes in blowing snow (or snow on ground). It also neglects the potential dependence of snow grain shapes on their size. Therefore, there is no guarantee that it represents the snow SSPs equally well at other wavelengths or for all snow grain sizes.
- Since absorption is very weak at $\lambda=0.80 \mu \mathrm{m}$, the observations do not constrain properly absorption by snow. Therefore, we cannot expect that our parameterization of $\beta$ (Eq. 11) predicts precisely the actual values for snow. However, we do expect that it captures reasonably the systematic difference between non-spherical snow grains and spheres: in general $\beta$ is larger for nonspherical particles.

- It is also possible that the snow grain shapes, and therefore the SSPs of snow on ground, might differ from those of blowing snow, and they might well vary from case to case, depending on how much metamorphosis the snow has experienced.

All these issues point to the need for validation of the derived parameterization against actual snow reflectance measurements in future work.

In spite of the concerns mentioned above, it seems reasonable to assume that the OHC selected here provides a substantially better basis for representing the SSPs of snow than spheres do. Moreover, the parameterization equations provided in this paper are analytic and simple to use. A Fortran implementation of the snow SSP parameterizations is available at https:/github.com/praisanen/snow_ssp.

To conclude, this paper describes a first-of-its-kind parameterization for representing the SSPs of snow in the solar spectral region. The parameterization is provided in hope that it will be useful, especially to those researchers that still use spherical particles for computing the radiative effects of snow. Nevertheless, it should definitely not be viewed as the "final solution" to the treatment of SSPs of snow. We hope that the present work will inspire the future development of snow SSP parameterizations based on more comprehensive data sets. Furthermore, at least in principle, it would be desirable to replace the current approach (where the shape distribution of snow grains is selected based on scattering measurements only) with an approach that more directly links the snow grain shapes to those actually observed. This would require, first, the parameterization of the size-shape distribution of snow grains based on observations and, second, the computation and parameterization of their SSPs. The main challenge in such an approach is the treatment of irregular grains, which are very common in snow. 


\section{Appendix A: Extrapolation of single-scattering properties}

The largest value of volume-to-projected area equivalent radius for which the SSPs are defined for aggregates of 10 plates in the Yang et al. (2013) database is $r_{\mathrm{vp}, \max }=653 \mu \mathrm{m}$, which falls below the upper limit of $2000 \mu \mathrm{m}$ considered for the OHC. Thus, to extend the SSPs for the OHC to sizes up to $r_{\mathrm{vp}}=2000 \mu \mathrm{m}$, we extrapolated the SSPs for the aggregates of plates based on how the SSPs depend on size for Koch fractals:

$$
\begin{gathered}
\begin{array}{c}
Q_{\text {ext, aggregate }}\left(r_{\mathrm{vp}}\right)=2+\left[Q_{\text {ext, aggregate }}\left(r_{\mathrm{vp}, \mathrm{lim}}\right)-2\right] \\
\cdot \frac{r_{\mathrm{vp}, \mathrm{lim}}}{r_{\mathrm{vp}}},
\end{array} \\
\beta_{\text {aggregate }}\left(r_{\mathrm{vp}}\right)=\beta_{\text {aggregate }}\left(r_{\mathrm{vp}, \lim }\right) \cdot \frac{\beta_{\text {fractal }}\left(r_{\mathrm{vp}}\right)}{\beta_{\text {fractal }}\left(r_{\mathrm{vp}, \mathrm{lim}}\right)},
\end{gathered}
$$

$$
\begin{gathered}
g_{\text {aggregate }}\left(r_{\mathrm{vp}}\right)=1-\left[1-g_{\text {aggregate }}\left(r_{\mathrm{vp}, \lim }\right)\right] \\
\cdot \frac{1-g_{\text {fractal }}\left(r_{\mathrm{vp}}\right)}{1-g_{\text {fractal }}\left(r_{\mathrm{vp}, \lim )}\right)}, \\
P_{11 \text {,aggregate }}\left(r_{\mathrm{vp}}, \theta_{\mathrm{s}}\right)=P_{11, \text { aggregate }}\left(r_{\mathrm{vp}, \lim }, \theta_{\mathrm{s}}\right) \\
\cdot \frac{P_{11 \text {,fractal }}\left(r_{\mathrm{vp}}, \theta_{\mathrm{s}}\right)}{P_{11, \text { fractal }}\left(r_{\left.\mathrm{vp}, \lim , \theta_{\mathrm{s}}\right)}\right.} .
\end{gathered}
$$

Here, $r_{\mathrm{vp}, \lim }=650 \mu \mathrm{m}$. While this is an ad hoc approach, the resulting uncertainty in the SSPs for the OHC (in which the aggregates of plates have a weight of $26 \%$ ) is most likely small. When the extrapolation was based on droxtals instead of Koch fractals, this changed the values of $g$ by at most 0.0025 and $\beta$ by at most 0.006 (or $1.4 \%$ in relative terms). 
Table A1. List of abbreviations and symbols.

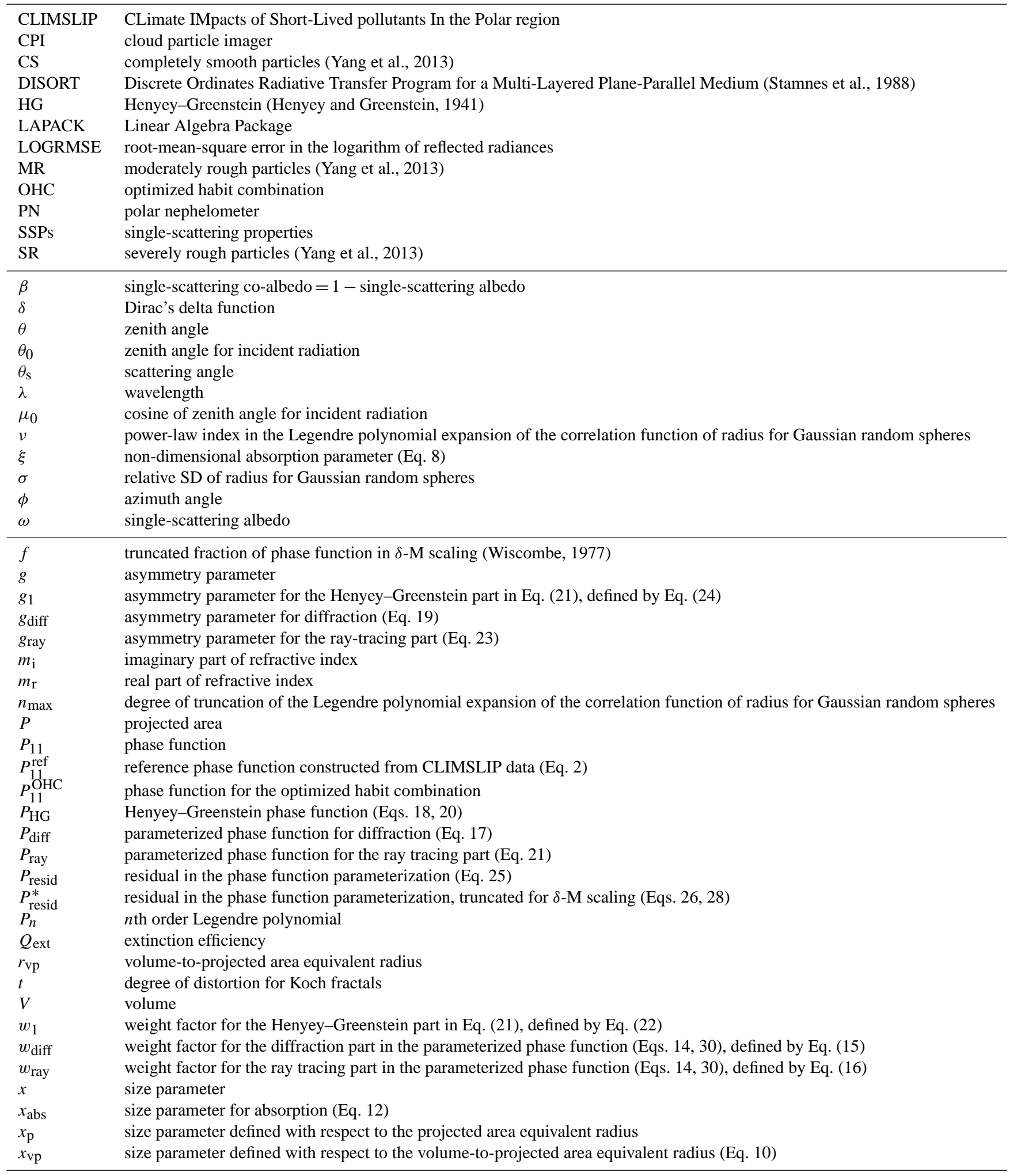


Acknowledgements. P. Räisänen was supported by the Nordic Centre of Excellence for Cryosphere-Atmosphere Interactions in a changing Arctic climate (CRAICC) and the Academy of Finland (grants nos. 140915 and 254195) and T. Nousiainen by the Academy of Finland (grant no. 255718) and the Finnish Funding Agency for Technology and Innovation (Tekes; grant no. 3155/31/2009). A. Kokhanovsky acknowledges the support of University of Bremen and project CLIMSLIP funded by BMBF. The CLIMSLIP field campaign was funded by the French Agence Nationale de la Recherche (ANR) and the Institut Polaire Français Paul Emile Victor (IPEV). We gratefully acknowledge the NILU and the Norsk Polarinstitutt for their technical assistance during the field campaign at Mount Zeppelin Station. Andreas Macke (Leibniz Institute for Tropospheric Research, Germany) is thanked for making available his ray tracing code. Ping Yang and Bingqi Yi (Texas AM University) are thanked for providing the Yang et al. (2013) database. Last but not least, Bastiaan van Diedenhoven and an anonymous referee are thanked for their helpful comments on the original manuscript.

Edited by: P. Marsh

\section{References}

Aoki, T., Aoki, T., Fukabori, M., Hachikubo, A., Tachibana, Y., and Nishio, F.: Effects of snow physical parameters on spectral albedo and bidirectional reflectance of snow surface, J. Geophys. Res., 105, 10219-10236, doi:10.1029/1999JD901122, 2000.

Aoki, T., Kuchiki, K., Niwano, M., Kodama, Y., Hosaka, M., and Tanaka, T.: Physically based snow albedo model for calculating broadband albedos and the solar heating profile in snowpack for general circulation models, J. Geophys. Res., 116, D11114, doi:10.1029/2010JD015507, 2011.

Barkey, B., Bailey, M., Liou, K.-N., and Hallett, J.: Light-scattering properties of plate and column ice crystals generated in a laboratory cold chamber, Appl. Optics, 41, 5792-5796, 2002.

Baum, B. A., Heymsfield, A. J., Yang, P., and Bedka, S. T., Bulk scattering properties for the remote sensing of ice clouds, Part I: Microphysical data and models, J. Appl. Meteorol., 44, 18851895, doi:10.1175/JAM2308.1, 2005.

Baum, B. A., Yang, P., Heymsfield, A. J., Schmitt, C. G., Xie, Y., Bansemer, A., Hu, Y.-X., and Zhang, Z.: Improvements in shortwave bulk scattering and absorption models for the remote sensing of ice clouds, J. Appl. Meteorol. Clim., 50, 1037-1056, doi:10.1175/2010JAMC2608.1, 2011.

Bi, L., Yang, P., Kattawar, G. W., and Kahn, R.: Single-scattering properties of triaxial ellipsoidal particles for a size parameter range from the Rayleigh to geometric-optics regimes, Appl. Optics, 48, 114-126, doi:10.1364/AO.48.000114, 2009.

Bohren, C. F. and Nevitt, T. J.: Absorption by a sphere: a simple approximation, Appl. Optics, 22, 774-775, 1983.

Crépel, O., Gayet, J.-F., Fournol, J.-F., and Oshchepkov, S.: A new airborne Polar Nephelometer for the measurement of optical and microphysical cloud properties, Part II: Preliminary tests, Ann. Geophys., 15, 460-470, doi:10.1007/s00585-997-0460-0, 1997.

de Rooij, W. A. and van der Stap, C. C. A. H.: Expansion of Mie scattering matrices in generalized spherical functions, Astron. Astrophys., 131, 237-248, 1984.
Dubovik, O., Sinuyk, A., Lapyonok, T., Holben, B. N., Mishchenko, M., Yang, P., Eck, T. F., Volten, H., Muñoz, O., Veihelmann, B., van der Zande, W. J., Leon, J.-F., Sorokin, M., and Slutsker, I.: Application of spheroid models to account for aerosol particle nonsphericity in remote sensing of desert dust, J. Geophys. Res., 111, D11208, doi:10.1029/2005JD006619, 2006.

Dubovik, O., Herman, M., Holdak, A., Lapyonok, T., Tanré, D., Deuzé, J. L., Ducos, F., Sinyuk, A., and Lopatin, A.: Statistically optimized inversion algorithm for enhanced retrieval of aerosol properties from spectral multi-angle polarimetric satellite observations, Atmos. Meas. Tech., 4, 975-1018, doi:10.5194/amt-4975-2011, 2011.

Durand, G., Gayet, J. F., Kaës, L., and Matharan, P.: Airborne infrared and microphysical measurements on cirrus clouds, in: Satellite Remote Sensing of Clouds and the Atmosphere III, P. Soc. Photo-Opt. Ins., 3495, 72-81, 1998.

Flanner, M. G. and Zender, C. S.: Snowpack radiative heating: influence on Tibetan Plateau climate, Geophys. Res. Lett., 32, L06501, doi:10.1029/2004GL022076, 2005.

Francis, P. N., Foot, J. S., and Baran, A. J.: Aircraft measurements of the solar and infrared radiative properties of cirrus and their dependence on ice crystal shape, J. Geophys. Res., 104, 3168531695, 1999.

Gardner, A. S. and Sharp, M. J.: A review of snow and ice albedo and the development of a new physically based broadband albedo parameterization, J. Geophys. Res., 115, F01009, doi:10.1029/2009JF001444, 2010.

Gayet, J. F., Crépel, O., Fournol, J. F., and Oshchepkov, S.: A new airborne polar Nephelometer for the measurements of optical and microphysical cloud properties, Part I: Theoretical design, Ann. Geophys., 15, 451-459, doi:10.1007/s00585-997-0451-1, 1997.

Gayet, J.-F., Asano, S., Yamazaki, A., Uchiyama, A., Sinyuk, A., Jourdan, O., and Auriol, F.: Two case studies of winter continental-type water and mixed-phase stratocumuli over the sea, 1. Microphysical and optical properties, J. Geophys. Res., 107, 4569, doi:10.1029/2001JD001106, 2002.

Grenfell, T. C., Warren, S. G., and Mullen, P. C.: Reflection of solar radiation by the Antarctic snow surface at ultraviolet, visible, and near-infrared wavelengths, J. Geophys. Res., 99, 18669-18684, doi:10.1029/94JD01484, 1994.

Guyot, G., Jourdan, O., Olofson, F., Schwarzenboeck, A., Gourbeyre, C., Febvre, G., Dupuy, R., Bernard, C., Tunved, P., Ancellet, G., Law, K., Wobrock, W., and Shcherbakov, V.: Ground based in situ measurements of arctic cloud microphysical and optical properties at Mount Zeppelin (Ny-Alesund, Svalbard), in: Proceedings of the EGU conference, EGU2015-14794, 13-17 April 2015, Vienna, Austria, http://meetingorganizer. copernicus.org/EGU2015/EGU2015-14794.pdf, last access: 1 June 2015.

Henyey, L. G. and Greenstein, J. L.: Diffuse radiation in the galaxy, Astrophys. J., 93, 70-83, 1941.

Hong, G., Yang P., Baum, B. A., Heymsfield, A. J., and Xu, K.-M.: Parameterization of shortwave and longwave radiative properties of ice clouds for use in climate models, J. Climate, 22, 62876312, doi:10.1175/2009JCLI2844.1, 2009.

Jin, Z., Charlock, T. P., Yang, P., Xie, Y., and Miller, W.: Snow optical properties for different particle shapes with application to snow grain size retrieval and MODIS/CERES radiance compar- 
ison over Antarctica, Remote Sens. Environ., 112, 3563-3581, 2008.

Jourdan, O., Oshchepkov, S., Shcherbakov, V., Gayet, J.-F., and Isaka, H.: Assessment of cloud optical parameters in the solar region: retrievals from airborne measurements of scattering phase functions, J. Geophys. Res., 108, 4572, doi:10.1029/2003JD003493, 2003.

Jourdan, O., Mioche, G., Garrett, T. J., Schwarzenböck, A., Vidot, J., Xie, Y., Shcherbakov, V., Yang, P., and Gayet, J.-F.: Coupling of the microphysical and optical properties of an Arctic nimbostratus cloud during the ASTAR 2004 experiment: implications for light-scattering modeling, J. Geophys. Res., 115, D23206, doi:10.1029/2010JD014016, 2010.

Kokhanovsky, A.: Spectral reflectance of solar light from dirty snow: a simple theoretical model and its validation, The Cryosphere, 7, 1325-1331, doi:10.5194/tc-7-1325-2013, 2013.

Kokhanovsky, A. A.: On light scattering in random media with large densely packed particles, J. Geophys. Res., 103, 60896096, 1998.

Kokhanovsky, A. A. and Nauss, T.: Satellite-based retrieval of ice cloud properties using a semianalytical algorithm, J. Geophys. Res., 110, D19206, doi:10.1029/2004JD005744, 2005.

Kokhanovsky, A. A. and Zege, E. P.: Scattering optics of snow, Appl. Optics, 43, 1589-1602, 2004.

Kokhanovsky, A. A., Aoki, T., Hachikubo, A., Hori, M., and Zege, E. P.: Reflective properties of natural snow: approximate asymptotic theory versus in situ measurements, IEEE Geosci. Remote S., 43, 1529-1535, 2005.

Kokhanovsky, A. A, Rozanov, V. V., Aoki, T., Odermatt, D., Brockmann, C., Krüger, O., Bouvet, M., Drusch, M., and Hori, M.: Sizing snow grains using backscattered solar light, Int. J. Remote Sens., 32, 6975-7008, 2011.

Lawson, R. P., Baker, B. A., Schmitt, C. G., and Jensen, T. L.: An overview of microphysical properties of Arctic clouds observed in May and July 1998 during FIRE ACE, J. Geophys. Res., 106, 14989-15014, doi:10.1029/2000JD900789, 2001.

Levy, R. C., Remer, L. A., and Dubovik, O.: Global aerosol optical properties and application to Moderate Resolution Imaging Spectroradiometer aerosol retrieval over land, J. Geophys. Res., 112, D13210, doi:10.1029/2006JD007815, 2007.

Lyapustin, A., Tedesco, M., Wang, Y., Aoki, T., Hori, M., and Kokhanovsky, A.: Retrieval of snow grain size over Greenland from MODIS, Remote Sens. Environ., 113, 1976-1987, 2009.

Macke, A.: Scattering of light by polyhedral ice crystals, Appl. Optics, 32, 2780-2788, 1993.

Macke, A., Müller, J., and Raschke, E.: Single scattering properties of atmospheric ice crystals, J. Atmos. Sci., 53, 2813-2825, 1996.

Mishchenko, M. I., Dlugach, J. M., Yanovitskij, E. G., and Zakharova, N. T.: Bidirectional reflectance of flat, optically thick particulate layers: an efficient radiative transfer solution and applications to snow and soil surfaces, J. Quant. Spectrosc. Ra., 63, 409-432, 1999.

Muinonen, K., Nousiainen, T., Fast, P., Lumme, K., and Peltoniemi, J. I.: Light scattering by Gaussian random particles: ray optics approximation, J. Quant. Spectrosc. Ra., 55, 577-601, 1996.

Negi, H. S. and Kokhanovsky, A.: Retrieval of snow albedo and grain size using reflectance measurements in Himalayan basin, The Cryosphere, 5, 203-217, doi:10.5194/tc-5-203-2011, 2011.
Nousiainen, T. and McFarquhar, G. M.: Light scattering by quasispherical ice crystals, J. Atmos. Sci., 61, 2229-2248, 2004.

Nousiainen, T. and Muinonen, K.: Light scattering by Gaussian, randomly oscillating raindrops, J. Quant. Spectrosc. Ra., 63, 643-666, 1999.

Nousiainen, T., Lindqvist, H., McFarquhar, G. M., and Um, J.: Small irregular ice crystals in tropical cirrus, J. Atmos. Sci., 68, 2614-2627, 2011.

Shcherbakov, V., Gayet, J.-F., Baker, B., and Lawson, P.: Light scattering by single natural ice crystals, J. Atmos. Sci., 63, 15131525, doi:10.1175/JAS3690.1, 2006.

Smith, H. R., Connolly, P. J., Baran, A. J., Hesse, E., Smedley, A. R. D., and Webb, A. R.: Cloud chamber laboratory investigations into scattering properties of hollow ice particles, J. Quant. Spectrosc. Ra., 157, 106-118, doi:10.1016/j.jqsrt.2015.02.015, 2015.

Stamnes, K., Tsay, S. C., Wiscombe, W., and Jayaweera, K.: Numerically stable algorithm for discrete-ordinate-method radiative transfer in multiple scattering and emitting layered media, Appl. Optics, 27, 2502-2509, 1988.

Tanikawa T., Aoki, T., Hori, M., Hachikubo, A., and Aniya, M.: Snow bidirectional reflectance model using non-spherical snow particles and its validation with field measurements, EARSeL eProceedings, 5, 137-145, 2006.

van Diedenhoven, B., Ackerman, A. S., Cairns, B., and Fridlind, A. M.: A flexible parameterization for shortwave optical properties of ice crystals, J. Atmos. Sci., 71, 1763-1782, doi:10.1175/JAS-D-13-0205.1, 2014.

Wang, Z. and Zeng, X.: Evaluation of snow albedo in land models for weather and climate studies, J. Appl. Meteorol. Clim., 49, 363-380, 2010.

Warren, S. G. and Brandt, R. E.: Optical constants of ice from the ultraviolet to the microwave: a revised compilation, J. Geophys. Res., 113, D14220, doi:10.1029/2007JD009744, 2008.

Wiscombe, W. J.: The delta-M method: rapid yet accurate radiative flux calculations for strongly asymmetric phase functions, J. Atmos. Sci., 34, 1408-1422, 1977.

Wiscombe, W. J. and Warren, S. G.: A model for the spectral albedo of snow, I: Pure snow, J. Atmos. Sci., 37, 2712-2733, 1980.

Xie, Y., Yang, P., Gao, B.-C., Kattawar, G. W., and Mishchenko, M. I.: Effect of ice crystal shape and effective size on snow bidirectional reflectance, J. Quant. Spectrosc. Ra., 100, 457-469, doi:10.1016/j.jqsrt.2005.11.056, 2006.

Yang, P. and Liou, K.-N.: Single-scattering properties of complex ice crystals in terrestrial atmospheres, Contrib. Atmos. Phys., 71, 223-248, 1998.

Yang, P., Bi, L., Baum, B. A., Liou, K.-N., Kattawar, G. W., Mishchenko, M. I., and Cole, B.: Spectrally consistent scattering, absorption and polarization of atmospheric ice crystals at wavelengths from 0.2 to $100 \mu \mathrm{m}$, J. Atmos. Sci., 70, 330-347, 2013.

Yurkin, M. A., Maltsev, V. P., and Hoekstra, A. G.: The discrete dipole approximation for simulation of light scattering by particles much larger than the wavelength, J. Quant. Spectrosc. Ra., 106, 546-557, 2007.

Zege, E. P., Katsev, I. L., Malinka, A. V., Prikhach, A. S., Heygster, G., and Wiebe, H.: Algorithm for retrieval of the effective snow grain size and pollution amount from satellite measurements, Remote Sens. Environ., 115, 2674-2685, 2011. 